\title{
Quantile Connectedness: Modeling Tail Behavior in the Topology of Financial Networks*
}

\author{
October 19, 2020
}

\begin{abstract}
We develop a new technique to estimate vector autoregressions with a common factor error structure by quantile regression. We apply our technique to study credit risk spillovers among a group of 17 sovereigns and their respective financial sectors between January 2006 and December 2017. We show that idiosyncratic credit risk shocks propagate much more strongly in both tails than at the conditional mean or median. Furthermore, we develop a measure of the relative spillover intensity in the right and left tails of the conditional distribution that provides a timely aggregate measure of systemic financial fragility and that can be used for risk management and monitoring purposes.
\end{abstract}

JEL Codes: C31, C32, C58, E44, G01.

KeYwords: Network Modeling; Quantile Vector Autoregression with Common Factors; Quantile Connectedness; Financial-Sovereign Credit Risk Transmission; Tail-Dependence.

${ }^{*}$ Declarations of interest: None.

Role of funding sources in the preparation of this manuscript: None. 


\section{Introduction}

The topology of financial networks is central to the study of systemic risk. An adverse idiosyncratic shock to one part of the financial system poses a threat to systemic stability if there are linkages through which it can propagate to other parts of the system. Measuring the nature and strength of financial market linkages is not only important for risk management strategies but also to inform the policy response to systemic crises. Several techniques for the econometric analysis of financial networks have been proposed in recent years, including those based on Granger-causality and on innovation accounting (e.g. Billio, Getmansky, Lo and Pelizzon, 2012; Diebold and Yilmaz, 2009, 2014; Alter and Beyer, 2014). A common feature of this literature is the reliance on conventional conditional mean estimators, such as ordinary least squares (OLS). The result is an estimate of the average network structure that prevails when an average shock affects the system. However, systemic shocks are likely to be much larger than average and it need not be the case that large shocks propagate in the same way as smaller shocks. To address this possibility, we develop a new framework that uses regression quantiles to investigate whether the topology of a network changes with the size of the shocks that affect the system.

Our framework builds upon that of Diebold and Yilmaz $(2009,2014)$, where the $m(m-1)$ bilateral interactions among an $m$-vector of variables, $\boldsymbol{y}_{t}$, are approximated by the $h$-steps-ahead forecast error variance decomposition (FEVD) of an underlying vector autoregression (VAR) in $\boldsymbol{y}_{t}$. Consequently, the Diebold-Yilmaz framework answers the question 'how much of the future uncertainty associated with variable $i$ can be attributed to shocks coming from variable $j$ ?'. A major advantage of the Diebold-Yilmaz framework relative to the Granger-causal network analysis of Billio et al. (2012) is that the resulting network is not only directed but also weighted and therefore provides an estimate of the strength of bilateral spillover effects. It has proven to be an influential technique, with applications including the connectedness among equity markets (e.g. Diebold and Yilmaz, 2009), foreign exchange markets (e.g. Baruník, Kočenda and Vácha, 2016; Greenwood-Nimmo, Nguyen and Rafferty, 2016), systemically important financial institutions (e.g. Demirer, Diebold, Liu and Yilmaz, 2018) and sovereign and corporate credit spreads (e.g. Greenwood-Nimmo, Huang and Nguyen, 2019; Bostanci and Yilmaz, 2020).

Rolling sample analysis is typically used to track the evolution of the network over time, with abrupt increases in connectedness often being interpreted in relation to systemic shocks. However, there is a tension between this interpretation and the fact that existing applications of the Diebold-Yilmaz framework rely on a range of conventional estimators, including OLS (e.g. 
Diebold and Yilmaz, 2009, 2014), the least absolute shrinkage and selection operator, or LASSO (e.g. Greenwood-Nimmo et al., 2019) and elastic net regularization (e.g. Demirer et al., 2018). Each of these estimators evaluates the relationship between $\boldsymbol{y}_{t}$ and $\boldsymbol{z}_{t}=\left\{\boldsymbol{y}_{t-1}, \boldsymbol{y}_{t-2}, \ldots, \boldsymbol{y}_{t-p}\right\}$ at the mean of the conditional distribution of $\boldsymbol{y}_{t} \mid \boldsymbol{z}_{t}$. The parameters of a VAR model estimated by OLS, for example, capture the dynamic relationships among the variables in $\boldsymbol{y}_{t}$ under the assumption that average shocks - that are precisely equal to zero by definition - affect the system. The tension arises because systemic shocks may often be larger than average. Consequently, studies in this literature implicitly assume that the relationships that prevail at the conditional mean can be generalized to the entire conditional distribution. This is a strong assumption but it is vital if rolling sample analysis based on conditional mean estimators is to provide a valid signal regarding the impact of large systemic shocks.

We relax this assumption by developing a new approach based on the premise that if one wishes to analyze the network structure associated with a large shock - for example a shock in the ninety-fifth percentile of the size distribution of shocks - then one must set aside conditional mean estimators and instead fit the VAR model at the ninety-fifth percentile by quantile regression. Following Koenker and Bassett (1978), quantile regression can be used to evaluate the dependence of $\boldsymbol{y}_{t}$ on $\boldsymbol{z}_{t}$ over the entire range of the conditional distribution of $\boldsymbol{y}_{t} \mid \boldsymbol{z}_{t}$. At the time of writing, two approaches to the estimation of quantile VAR models have been proposed by Cecchetti and Li (2008) and Schüler (2014), respectively. ${ }^{1}$ Cecchetti and Li develop an equation-by-equation estimation framework for VAR models with cross-sectionally correlated residuals, while Schüler develops a Bayesian framework for the analysis of structural VARs.

Our framework is distinct from these existing methods by virtue of our treatment of the VAR residuals. We assume that the cross-sectional correlation of the VAR residuals is driven by a finite number of unobserved common factors. This assumption serves two purposes. First, by purging the common component from the VAR residuals, we are able to isolate the idiosyncratic shock to each variable in the system. Not only does this align our framework with the large literature on systematic vs. idiosyncratic variations in finance - an important consideration if one wishes to use network models to analyze the propagation of idiosyncratic shocks, for example — but it also addresses the likelihood that the failure to account for sources of common variation may generate substantial biases in the analysis of networks. Specifically, if an omitted common

\footnotetext{
${ }^{1}$ The method of Cecchetti and Li (2008) has subsequently been applied by Linnemann and Winkler (2016) and Zhu, Su, Guo and Ren (2016). A related paper by Chavleishvili and Manganelli (2016) develops a framework for quantile impulse response analysis of a bivariate system with one endogenous and one exogenous variable.
} 
factor affects all variables in the VAR system, then the proportion of the forecast error variance that should rightly be attributed to that factor must instead be attributed to one or more of the endogenous variables in the system, thereby upwardly biasing the estimated spillover effects. ${ }^{2}$ The issue of spurious Granger causality arising due to the omission of sources of common variation is well-known but the impact of omitted common factors on Diebold-Yilmaz networks has not been adequately explored to date (see Claeys and Vašíček, 2014, for an example of a Diebold-Yilmaz network with common factors). Second, the use of a factor structure simplifies the estimation problem substantially because it attenuates the cross-section correlation among the VAR residuals, greatly simplifying the task of defining regression quantiles.

Our approach answers a modified version of the question addressed by the Diebold-Yilmaz framework: 'how much of the future uncertainty associated with variable $i$ can be attributed to idiosyncratic shocks coming from variable $j$ as the shock size varies?'. Consequently, our technique is ideally suited to the study of the propagation of idiosyncratic risk shocks and contagion, the latter of which is often defined in relation to the difference in the way that shocks propagate during rare events relative to normal times (e.g. Caporin, Pelizzon, Ravazzolo and Rigobon, 2018). We introduce the term quantile connectedness to distinguish between our quantile-based approach to network analysis and the established mean-based approach.

We apply our technique to study spillovers of idiosyncratic credit risk between sovereigns and national financial sectors over the period January 2006 to December 2017, both within and across borders. The study of credit risk transmission has become an important focus among policy institutions, with a particular concern for the emergence of feedback loops and destabilizing spirals (e.g. International Monetary Fund, 2013, pp. 65-6). Acharya, Drechsler and Schnabl (2014) provide compelling evidence of just such an adverse feedback effect. They demonstrate that the financial sector bailouts undertaken by many developed countries in 2008 amounted to a substantial transfer of private sector credit risk onto the public sector at a time of rapid public debt growth. This combination ultimately proved untenable in several countries and led to a resurgence of systemic risk driven by the emergence of an adverse feedback loop between sovereign credit risk and financial sector credit risk. Variants of the Diebold-Yilmaz technique have been applied to the analysis of the sovereign-financial credit risk network by Alter and Beyer (2014) and Greenwood-Nimmo et al. (2019), although our paper represents the

\footnotetext{
${ }^{2}$ In this paper, we focus on analyzing direct spillovers of idiosyncratic credit risk, having controlled for common systematic factors. Although it is not our focus here, one may also be interested in analyzing the indirect propagation of shocks via the common factors. The Diebold-Yilmaz framework accounts for both direct and indirect linkages but it does not allow them to be analyzed separately.
} 
first attempt to study quantile-variation in the structure of the credit risk network.

We follow the existing literature and use credit default swap (CDS) spreads to measure credit risk. $^{3}$ The existence of a factor structure in the cross-section of CDS spreads has been documented by Pan and Singleton (2008), Longstaff, Pan, Pedersen and Singleton (2011), Fender, Hayo and Neuenkirch (2012) and Ang and Longstaff (2013), among others. To isolate the idiosyncratic variation in the CDS spreads, our model accounts for an unobserved factor structure following Ando and Bai (2020), where the optimal number of unobserved common factors at a given conditional quantile is chosen by minimization of an information criterion. Ex post analysis of the common factors reveals that they are correlated with a number of observable variables, including various iTraxx credit risk indices, the US equity market excess return and the US equity variance risk premium. This is consistent with Longstaff et al. (2011), who note that sovereign CDS spreads exhibit strong correlations with US stock market returns and their implied volatility.

Our first finding is that the topology of the credit risk network varies substantially with the shock size over the full sample. We find that the effects of small idiosyncratic credit risk shocks in the central $40 \%$ of the conditional distribution are predominantly localized. Bilateral spillovers account for roughly $40 \%$ of the five-days-ahead forecast error variance (FEV) over this range. However, this is not true of large shocks. Large adverse shocks in the right tail of the conditional distribution spread forcefully through the financial system, with bilateral spillovers accounting for $79.36 \%$ and $91.77 \%$ of FEV at the ninety-fifth and ninety-ninth conditional quantiles, respectively. This finding accords with the existing evidence of increased financial market comovements under adverse conditions (e.g. Ang and Bekaert, 2002). Interestingly, we also find evidence that large beneficial shocks propagate strongly, with bilateral spillovers accounting for $77.17 \%$ and $88.18 \%$ of FEV at the fifth and first conditional quantiles, respectively. The finding that large shocks in both tails spill-over strongly is consistent with the existing literature on good and bad contagion (e.g. Jorion and Zhang, 2007; Londono, 2019). Crucially, when the

\footnotetext{
${ }^{3}$ A CDS contract operates like an insurance agreement negotiated between two parties, for example, one of whom holds a risky bond and the other of whom agrees to absorb losses arising should the bond issuer suffer a designated credit event, such as default. The CDS spread defines the price that the bondholder must pay to the protection seller. Blanco, Brennan and Marsh (2005) and Gyntelberg, Hördahl, Ters and Urban (2013) show that the CDS market is the leading forum for credit risk price discovery, providing more timely signals of changes in the credit risk environment than bond yield spreads. In addition, CDS spreads may offer a cleaner measure of credit risk than bond yield spreads, because the latter are directly affected by inflation. Losses associated with single name CDS contracts played an important role in the global financial crisis and sovereign CDS contracts were widely used for hedging and speculation during the European debt crisis. Although the CDS market has subsequently evolved due to regulation and has seen a notable reduction in outstanding notional value associated with the rise of index hedging strategies (Aldasoro and Ehlers, 2018), it remains an important forum for determining the price of credit risk.
} 
model is estimated at the conditional mean by OLS, this quantile variation is averaged out and bilateral spillovers are found to account for $56.57 \%$ of FEV. This result arises because OLS is equivalent to an equally-weighted average of quantile regression estimators over the entire conditional distribution. This cautions against the use of network models estimated using conditional mean estimators to analyze the spillovers associated with extreme events.

Our second result is that, over the full sample, the adverse feedback effect documented by Acharya et al. (2014) and Farhi and Tirole (2018) manifests as a marked intensification of the bidirectional feedback between each sovereign and its domestic financial sector in the presence of large adverse shocks in the right tail of the conditional distribution. However, we once again find that this behavior is not unique to adverse shocks - there is a similar intensification of bidirectional feedback in the left tail. This leads us to conclude that the adverse feedback loop that generates a vicious cycle by amplifying bad news is counterbalanced to some extent by the existence of a virtuous feedback loop that can rapidly reduce credit spreads when beneficial shocks occur.

Our final result is derived from rolling sample estimation of our model. In this way, we demonstrate that the dependence structure that exists among the cross-section of sovereigns and financial institutions displays marked time-variation. The time-variation of bilateral credit risk spillovers has already been demonstrated at the conditional mean (e.g. Greenwood-Nimmo et al., 2019). However, we are the first to demonstrate that the time-variation in the network topology observed in the tails of the conditional distribution does not closely resemble the time-variation observed at either the conditional mean or median. This is an important finding, given the relevance of tail-dependence for financial supervision and risk management (e.g. Betz, Hautsch, Peltonen and Schienle, 2016) and it suggests that the implications derived from network models evaluated by conventional conditional mean estimators cannot necessarily be generalized to the tails. We show that major adverse events are associated with an increase in average connectedness but that their effects on the tails differs. Specifically, we find that right-tail-dependence increases markedly but that left-tail-dependence often does not respond strongly and may even decrease. By contrast, stabilizing policy interventions that reduce average connectedness tend to increase left-tail-dependence but may not exert a strong influence on right-tail-dependence. We suggest that the differential behavior of right- and lefttail-dependence may arise from the aggregate actions of market participants if the information revealed by a major event in either tail causes a non-trivial proportion of market participants 
to focus disproportionately on further events occurring in that tail while paying less attention to events in the other tail.

We develop a new measure of relative tail-dependence denoted $R T D$, which draws attention to the differential behavior of left- and right-tail-dependence. As a tail-based aggregate measure of financial fragility, $R T D$ bears a non-trivial resemblance to the CATFIN index proposed by Allen, Bali and Tang (2012). CATFIN is an aggregate downside risk measure obtained as an equally-weighted average of the $99 \%$ value-at-risk (VaR) estimated in three different ways. However, unlike CATFIN, RTD is a not a downside risk measure but a relative measure that exploits information from both tails of the conditional distribution. In practice, $R T D$ tracks CATFIN relatively closely over our sample period, with the two sharing a correlation of 0.42 and exhibiting similar turning points. Given that $R T D$ captures a related but subtly different phenomenon than CATFIN, we argue that the two are complementary and provide policymakers and practitioners alike with a valuable indication of aggregate changes in systemic risk.

Aside from the work on empirical network modeling, we wish to highlight four strands of literature to which our paper is related. First, our use of quantile regression to study extreme events closely resembles a branch of the systemic risk literature that is well-represented by Betz et al. (2016) and Caporin et al. (2018), both of which use quantile regression to study the propagation of adverse shocks through the financial markets. Second, our concept of timevarying quantile connectedness is complementary to the extreme value theory (EVT) literature on time-varying tail dependence (e.g. McNeil and Frey, 2000; Bali and Weinbaum, 2007), with each technique offering particular benefits. An appealing feature of the EVT approach is that it offers a parametric form for the tail of a distribution, thereby providing a basis for extrapolation beyond the range of the data. However, the theory underlying the application of EVT methods to multivariate systems with cross-section and serial dependence is yet to be adequately developed. Our quantile connectedness framework enjoys an advantage in this respect, as it can accommodate both cross-section and serial dependence through the factor structure. Third, our work has a natural link to VaR and associated concepts, such as CoVaR (Adrian and Brunnermeier, 2016) and CATFIN (Allen et al., 2012). VaR is widely used by investors to measure the potential loss that they may endure on their positions due to adverse shocks over a defined horizon and at a predetermined confidence level. To illustrate the conceptual link, assume that investors maintain sufficient capital reserves to cover the VaR at the 95\% confidence level. In this case, losses up to the VaR can be absorbed within the capital buffer. 
However, the probability of an adverse shock sufficiently severe to generate a loss in excess of the VaR is $5 \%$. Investors may find the losses caused by such large shocks untenable, raising the possibility of default and insolvency. As a result, the transmission of risk among counterparties may be considerably stronger in the case of large shocks than small shocks. This offers a partial explanation of the quantile-variation that we document in the topology of the credit risk network. Finally, the notion that the topology of a network may vary with the size of the shocks affecting the system is related to Acemoglu, Ozdaglar and Tahbaz-Salehi's (2015) insight that a phase change may occur, whereby dense financial networks are resilient to small shocks but can be vulnerable to cascading failures in the event of a large adverse shock.

This paper proceeds as follows. In Section 2, we outline the quantile factor VAR model and derive the associated FEVD, which is then used to construct network statistics. We discuss the construction and properties of our dataset in Section 3, before presenting our estimation results in Section 4. We conclude in Section 5. Further information regarding our dataset and a brief synopsis of two substantial robustness exercises are provided in four appendices.

\section{Quantile Connectedness}

In this section, we propose a new framework for the graphical analysis of VAR models estimated at a given conditional quantile, $\tau \in(0,1)$. To this end, we develop a framework for the equationby-equation estimation of a VAR model by quantile regression, where a factor structure is used to distinguish between the common and idiosyncratic components of the error process. We then derive the associated $h$-steps-ahead FEVD, which forms the basis for network analysis in the tradition of Diebold and Yilmaz (2009, 2014).

\subsection{The Quantile VAR Model}

Consider a multi-country environment, where countries are indexed by $i=1,2, \ldots, N$ and time periods are indexed by $t=1,2, \ldots T$. Let $\boldsymbol{y}_{i t}=\left(\Delta s_{i t}, \Delta f_{i t}\right)^{\prime}$, where $s_{i t}$ and $f_{i t}$ denote the sovereign and financial sector CDS spreads for the $i$ th country, which will be discussed in detail below. Moreover, let the $m \times 1$ vector $\boldsymbol{y}_{t}=\left(\boldsymbol{y}_{1 t}^{\prime}, \boldsymbol{y}_{2 t}^{\prime}, \ldots, \boldsymbol{y}_{N t}^{\prime}\right)^{\prime}$ collect the credit spreads for all $N$ countries in the system, where $m=2 N$. Implementation of the Diebold-Yilmaz technique requires the specification of a model capturing the dynamics of $\boldsymbol{y}_{t}$. To this end, consider the following VAR model, which expresses the sovereign and financial sector CDS spreads for the $i$ th country as a function of the lagged sovereign and financial sector CDS spreads for every 
country in the system:

$$
\boldsymbol{y}_{i t}=\boldsymbol{\mu}_{i}+\sum_{j=1}^{p} \boldsymbol{\Phi}_{i j} \boldsymbol{y}_{t-j}+\boldsymbol{e}_{i t},
$$

where $\underset{2 \times 1}{\boldsymbol{\mu}_{i}}$ is a vector of intercepts, $\underset{2 \times m}{\boldsymbol{\Phi}_{i j}}$ is the parameter matrix at the $j$ th lag and the regression residuals $\underset{2 \times 1}{\boldsymbol{e}_{i t}} \sim\left(\mathbf{0}, \boldsymbol{\Sigma}_{\boldsymbol{i}}\right)$, where $\underset{2 \times 2}{\boldsymbol{\Sigma}_{\boldsymbol{i}}}$ is a positive definite covariance matrix. By stacking (1) for all countries in the system, we obtain the following VAR system for $\boldsymbol{y}_{t}$ :

$$
\boldsymbol{y}_{t}=\boldsymbol{\mu}+\sum_{j=1}^{p} \boldsymbol{\Phi}_{j} \boldsymbol{y}_{t-j}+\boldsymbol{e}_{t}
$$

where $\underset{m \times 1}{\boldsymbol{\mu}}=\left(\boldsymbol{\mu}_{1}^{\prime}, \boldsymbol{\mu}_{2}^{\prime}, \ldots, \boldsymbol{\mu}_{N}^{\prime}\right)^{\prime}$ is a vector of intercepts, $\underset{m \times m}{\mathbf{\Phi}_{j}}=\left(\boldsymbol{\Phi}_{1 j}^{\prime}, \boldsymbol{\Phi}_{2 j}^{\prime}, \ldots, \boldsymbol{\Phi}_{N j}^{\prime}\right)^{\prime}$ is the $j$ th autoregressive parameter matrix and the residual process $\underset{m \times 1}{\boldsymbol{e}_{t}}=\left(\boldsymbol{e}_{1 t}^{\prime}, \boldsymbol{e}_{2 t}^{\prime}, \ldots, \boldsymbol{e}_{N t}^{\prime}\right)^{\prime} \sim(\mathbf{0}, \boldsymbol{\Sigma})$, where $\underset{m \times m}{\boldsymbol{\Sigma}}$ is positive definite.

The order of the VAR model, $p$, can be consistently estimated using the Schwarz Information Criterion. Nonetheless, the residuals will typically exhibit contemporaneous cross-section correlation and so $\boldsymbol{\Sigma}$ is likely to be non-diagonal. ${ }^{4}$ Conditional mean estimation of unrestricted VARs of this type is straightforward, and can be achieved on an equation-by-equation basis using OLS. Conditional quantile estimation is more challenging, however. Given that each equation in (2) shares a common set of right hand side variables, the estimation problem has a seemingly unrelated regressions (SUR) structure that has led several authors to pursue an equation-by-equation quantile regression estimation strategy (e.g. Cecchetti and Li, 2008; Linnemann and Winkler, 2016; Zhu et al., 2016). However, from equation (5) in Zellner and Ando (2010), it is clear that this approach sets the off-diagonal elements of the covariance matrix of $\boldsymbol{e}_{t}$ to zero, which amounts to the assumption of cross-section independence. The failure to adequately account for cross-section correlation among the regression residuals is likely to bias the resulting parameter estimates.

We address this issue by modeling the cross-section correlation in the residuals as the result of an $f \times 1$ vector of common factors. In this way, we are able to separate the systematic and idiosyncratic components of the error process, thereby aligning our approach with the large literature on the distinction between systematic and idiosyncratic risks (see Feldhütter and Nielsen, 2012, for an example focusing on credit spreads). Furthermore, where one wishes to

\footnotetext{
${ }^{4}$ When using VARs for macroeconomic analysis, it is common to transform the reduced form VAR (2) into a structural counterpart with uncorrelated disturbance terms to which one can attach a structural interpretation. This is typically achieved either by imposing Wold-causality as in Sims (1986), short-run exclusion restrictions as in Blanchard and Watson (1986) or long-run restrictions as in Blanchard and Quah (1989). However, the application of these traditional methods becomes increasingly challenging as the dimension of the model increases.
} 
analyze spillover effects between variables, it is important to focus on the idiosyncratic variation having purged any systematic variation, or else one is likely to obtain a biased estimate of the spillover intensity (see Claeys and Vašíček, 2014, for a related discussion). Formally, we assume that:

$$
\boldsymbol{e}_{i t}=\boldsymbol{\lambda}_{i}^{\prime} \boldsymbol{f}_{t}+\boldsymbol{v}_{i t}
$$

and, by extension, that:

$$
\boldsymbol{e}_{t}=\boldsymbol{\Lambda} \boldsymbol{f}_{t}+\boldsymbol{v}_{t}
$$

where $\underset{f \times 1}{\boldsymbol{f}_{t}}$ is a vector of common factors, $\underset{m \times f}{\boldsymbol{\Lambda}}=\left(\boldsymbol{\lambda}_{1}^{\prime}, \boldsymbol{\lambda}_{2}^{\prime}, \ldots, \boldsymbol{\lambda}_{N}^{\prime}\right)^{\prime}$ is a matrix of heterogeneous factor loadings and $\underset{m \times 1}{\boldsymbol{v}_{t}}=\left(\boldsymbol{v}_{1 t}^{\prime}, \boldsymbol{v}_{2 t}^{\prime}, \ldots, \boldsymbol{v}_{N t}^{\prime}\right)^{\prime} \sim(\mathbf{0}, \boldsymbol{\Omega})$ contains the idiosyncratic components of $\boldsymbol{e}_{t}$. Combining (2) and (4) yields the following factor VAR model:

$$
\boldsymbol{y}_{t}=\boldsymbol{\mu}+\sum_{j=1}^{p} \boldsymbol{\Phi}_{j} \boldsymbol{y}_{t-j}+\boldsymbol{\Lambda} \boldsymbol{f}_{t}+\boldsymbol{v}_{t}
$$

where $\boldsymbol{f}_{t}$ is a vector of unobserved common factors and $\boldsymbol{\Lambda}$ denotes the corresponding factor loadings. Estimation of and inference on models of this type at the conditional mean has been studied by Bai, Li and Lu (2016). In this paper, we are primarily interested in estimating the factor VAR model by quantile regression. The quantile function for $y_{i t}$ at the $\tau$-th conditional quantile is:

$$
Q_{\tau}\left(y_{i t} \mid \mathcal{F}_{t-1}\right)=\mu_{i,(\tau)}+\sum_{j=1}^{p} \phi_{i, j,(\tau)}^{\prime} \boldsymbol{y}_{t-j}+\boldsymbol{\lambda}_{i,(\tau)} \boldsymbol{f}_{t,(\tau)},
$$

where $\mathcal{F}_{t-1}$ denotes the information set available at time $t-1, \mu_{i,(\tau)}$ is the $i$-th element of $\boldsymbol{\mu}_{(\tau)}=\left(\mu_{1,(\tau)}, \ldots, \mu_{m,(\tau)}\right)^{\prime}, \boldsymbol{\phi}_{i, j(\tau)}$ and $\boldsymbol{\lambda}_{i,(\tau)}$ are the $i$-th rows of $\boldsymbol{\Phi}_{j(\tau)}$ and $\boldsymbol{\Lambda}_{(\tau)}$, respectively, and $\boldsymbol{f}_{t,(\tau)}$ is the vector of unobserved common factors at the $\tau$-th conditional quantile, with dimension $f_{(\tau)}$. We follow the precedent of Koenker and Xiao (2006) and assume that the optimal lag order for the conditional mean model remains valid at every conditional quantile.

The unknown parameters to be estimated are $\boldsymbol{\mu}_{(\tau)}, \boldsymbol{\Phi}_{1(\tau)}, \ldots, \boldsymbol{\Phi}_{p(\tau)}, \boldsymbol{F}_{(\tau)}$ and $\boldsymbol{\Lambda}_{(\tau)}$, where $\boldsymbol{F}_{(\tau)}=\left(\boldsymbol{f}_{1,(\tau)}, \ldots, \boldsymbol{f}_{T,(\tau)}\right)^{\prime}$. For a fixed value of $\tau$ and a fixed number of common factors, these unknown parameters can be estimated as follows:

$$
\min _{\boldsymbol{\mu}_{(\tau)}, \boldsymbol{\Phi}_{1(\tau)}, \ldots, \mathbf{\Phi}_{p(\tau)}, \boldsymbol{F}_{(\tau)}, \boldsymbol{\Lambda}_{(\tau)}} \frac{1}{m T} \sum_{i=1}^{m} \sum_{t=1}^{T} \xi_{\tau}\left(y_{i t}-\mu_{i,(\tau)}-\sum_{j=1}^{p} \boldsymbol{\phi}_{i, j,(\tau)}^{\prime} \boldsymbol{y}_{t-j}-\boldsymbol{\lambda}_{i,(\tau)} \boldsymbol{f}_{t,(\tau)}\right)
$$

where $\xi_{\tau}(z)$ is the check loss function defined as $\xi_{\tau}(z)=z\left(\tau-1_{[z \leq 0]}\right)$ as in Koenker and Hallock 
(2001). Ando and Bai (2020) establish the consistency and asymptotic normality of the resulting parameter estimates under mild regularity conditions.

For identification purposes, normalization restrictions are imposed on $\boldsymbol{F}_{(\tau)}$ and $\boldsymbol{\Lambda}_{(\tau)}$. Following Bai and $\mathrm{Ng}(2013)$, we use the restrictions $\boldsymbol{F}_{(\tau)}^{\prime} \boldsymbol{F}_{(\tau)} / T=I_{f_{(\tau)}}$ and $\boldsymbol{\Lambda}_{(\tau)}^{\prime}=\left(\boldsymbol{\Lambda}_{1,(\tau)}^{\prime}, \boldsymbol{\Lambda}_{2,(\tau)}^{\prime}\right)^{\prime}$, with $\boldsymbol{\Lambda}_{1,(\tau)}$ being an invertible lower triangular matrix. ${ }^{5}$ Following Ando and Bai (2020), the number of common factors can be selected by minimizing the following information criterion:

$$
\begin{aligned}
\min _{f_{(\tau)}}[\log & \left(\frac{1}{m T} \sum_{i=1}^{m} \sum_{t=1}^{T} \xi_{\tau}\left(y_{i t}-\hat{\mu}_{i,(\tau)}-\sum_{j=1}^{p} \hat{\boldsymbol{\phi}}_{i, j,(\tau)}^{\prime} \boldsymbol{y}_{t-j}-\hat{\boldsymbol{\lambda}}_{i,(\tau)} \hat{\boldsymbol{f}}_{t,(\tau)}\right)\right) \\
& \left.+f_{(\tau)} \times\left(\frac{m+T}{m T}\right) \log \left(\frac{m T}{m+T}\right)\right]
\end{aligned}
$$

where $\hat{\mu}_{i,(\tau)}, \hat{\boldsymbol{\phi}}_{i, j,(\tau)}, \hat{\boldsymbol{\lambda}}_{i,(\tau)}$ and $\hat{\boldsymbol{f}}_{t,(\tau)}$ are the estimated parameters of (7) for a given number of factors.

\subsection{Quantile Forecast Error Variance Decomposition}

The functional form of the factor VAR model (5) is identical to a $\operatorname{VARX}(p, 0)$ model - that is, a VAR model with $p$ lags of a set of endogenous variables augmented with the contemporaneous values of a set of exogenous variables. Two approaches to innovation accounting with VARX models have been pursued in the literature: (i) to conduct innovation accounting by conditioning on the values of the exogenous variables; and (ii) to augment the VARX specification with an auxiliary marginal model for the exogenous variables and then to undertake innovation accounting with respect to the augmented system. Given that our interest is in modeling the propagation of the idiosyncratic shocks as opposed to the effects of systemwide shocks, we pursue the former option. In addition, as the computation of the FEVD for VARX models evaluated at the conditional mean is well established (e.g. Garratt, Lee, Pesaran and Shin, 2006), we limit our attention to the quantile factor VAR (QFVAR) case.

We start with the following QFVAR model:

$$
\boldsymbol{y}_{t}=\sum_{j=1}^{p} \boldsymbol{\Phi}_{j(\tau)} \boldsymbol{y}_{t-j}+\boldsymbol{\Lambda}_{(\tau)}^{*} \boldsymbol{f}_{t(\tau)}^{*}+\boldsymbol{v}_{t(\tau)}
$$

where $\boldsymbol{\Lambda}_{(\tau)}^{*}=\left(\boldsymbol{\mu}_{(\tau)}, \boldsymbol{\Lambda}_{(\tau)}\right), \boldsymbol{f}_{t(\tau)}^{*}=\left(1, \boldsymbol{f}_{t(\tau)}^{\prime}\right)^{\prime}$. The Wold representation of (9) can be written

\footnotetext{
${ }^{5}$ The reader is referred to Bai and $\mathrm{Ng}(2002,2013)$ for details of the principal component estimator for the conditional mean model.
} 
as:

$$
Q_{\tau}\left(\boldsymbol{y}_{t} \mid \mathcal{F}_{t-1}\right)=\sum_{j=0}^{\infty} \boldsymbol{B}_{j(\tau)} \boldsymbol{v}_{t-j(\tau)}+\sum_{j=0}^{\infty} \boldsymbol{C}_{j(\tau)} \boldsymbol{f}_{t-j(\tau)}^{*},
$$

where $\boldsymbol{B}_{j(\tau)}=\boldsymbol{\Phi}_{1(\tau)} \boldsymbol{B}_{j-1(\tau)}+\boldsymbol{\Phi}_{2(\tau)} \boldsymbol{B}_{j-2(\tau)}+\ldots$ for $j=1,2, \ldots$ with $\boldsymbol{B}_{0(\tau)}=\boldsymbol{I}_{m}$ and $\boldsymbol{B}_{j(\tau)}=\mathbf{0}$ for $\ell<0$ and where $\boldsymbol{C}_{j(\tau)}=\boldsymbol{B}_{j(\tau)} \boldsymbol{\Lambda}_{(\tau)}^{*}$.

Following the precedent established by Cecchetti and Li (2008, p. 12) in the context of multivariate forecasting with dynamic quantile regressions, we assume that the quantile index is fixed throughout the forecast horizon. ${ }^{6}$ Under this assumption, (10) implies that the vector of forecast errors associated with the prediction of $\boldsymbol{y}_{t+h}$ conditional on the information available at time $t-1$ and on the common factors is given by:

$$
\boldsymbol{u}_{t+h(\tau)}=\sum_{\ell=0}^{h} \boldsymbol{B}_{\ell(\tau)} \boldsymbol{v}_{t+h-\ell(\tau)}
$$

and the total forecast error variance matrix is:

$$
\operatorname{Cov}\left(\boldsymbol{u}_{t+h(\tau)}\right)=\sum_{\ell=0}^{h} \boldsymbol{B}_{\ell(\tau)} \boldsymbol{\Omega}_{(\tau)} \boldsymbol{B}_{\ell(\tau)}^{\prime} .
$$

Now, consider the covariance matrix of the forecast errors associated with predicting $\boldsymbol{y}_{t+h}$ given values of the shocks to the $i$ th equation, $v_{i t(\tau)}, v_{i, t+1(\tau)}, \ldots, v_{i, t+h(\tau)}$ :

$$
\boldsymbol{u}_{t+h(\tau)}^{(i)}=\sum_{\ell=0}^{h} \boldsymbol{B}_{\ell(\tau)}\left(\boldsymbol{v}_{t+h-\ell(\tau)}-E\left(\boldsymbol{v}_{t+h-\ell(\tau)} \mid v_{i, t+h-\ell(\tau)}\right)\right) .
$$

Now, we have:

$$
\begin{aligned}
E\left(\boldsymbol{v}_{t+h-\ell(\tau)} \mid v_{i, t+h-\ell(\tau)}\right) & =\left(\omega_{i i,(\tau)}^{-1} \boldsymbol{\Omega}_{(\tau)} \mathbf{e}_{i}\right) v_{i, t+h-\ell(\tau)} \\
& =\mathbf{e}_{i} v_{i, t+h-\ell(\tau)}
\end{aligned}
$$

where $\mathbf{e}_{i}$ is an $m \times 1$ selection vector with its $i$ th element set to 1 and all other elements set to zero and where $\omega_{i i,(\tau)}^{-1} \boldsymbol{\Omega}_{(\tau)} \mathbf{e}_{i}=\mathbf{e}_{i}$. Substituting (13) into (12), we obtain:

$$
\boldsymbol{u}_{t+h(\tau)}^{(i)}=\sum_{\ell=0}^{h} \boldsymbol{B}_{\ell(\tau)}\left(\boldsymbol{v}_{t+h-\ell(\tau)}-\left(\omega_{i i,(\tau)}^{-1} \boldsymbol{\Omega}_{(\tau)} \mathbf{e}_{i}\right) v_{i, t+h-\ell(\tau)}\right),
$$

\footnotetext{
${ }^{6}$ In principle, one could allow for the quantile index to vary across the forecast horizon, although it is not clear how one could systematically determine the time-path of the quantile index from one horizon to the next.
} 
and taking the unconditional expectation yields:

$$
\operatorname{Cov}\left(\boldsymbol{u}_{t+h(\tau)}^{(i)}\right)=\sum_{\ell=0}^{h} \boldsymbol{B}_{\ell(\tau)} \boldsymbol{\Omega}_{(\tau)} \boldsymbol{B}_{\ell(\tau)}^{\prime}-\omega_{i i,(\tau)}^{-1} \sum_{\ell=0}^{h} \boldsymbol{B}_{\ell(\tau)} \boldsymbol{\Omega}_{(\tau)} \mathbf{e}_{i} \mathbf{e}_{i}^{\prime} \boldsymbol{\Omega}_{(\tau)} \boldsymbol{B}_{\ell(\tau)}^{\prime} .
$$

Therefore, the decline in the $h$-steps-ahead forecast error variance of $\boldsymbol{y}_{t}$ obtained as a result of conditioning on future shocks to the $i$ th equation is given by:

$$
\begin{aligned}
\boldsymbol{\Delta}_{i h(\tau)} & =\operatorname{Cov}\left(\boldsymbol{u}_{t+h(\tau)}-\boldsymbol{u}_{t+h(\tau)}^{(i)}\right) \\
& =\omega_{i i,(\tau)}^{-1} \sum_{\ell=0}^{h} \boldsymbol{B}_{\ell(\tau)} \boldsymbol{\Omega}_{(\tau)} \mathbf{e}_{i} \mathbf{e}_{i}^{\prime} \boldsymbol{\Omega}_{(\tau)} \boldsymbol{B}_{\ell(\tau)}^{\prime}
\end{aligned}
$$

Scaling the $j$ th diagonal element of $\boldsymbol{\Delta}_{i h(\tau)}$ - that is, $\mathbf{e}_{j}^{\prime} \boldsymbol{\Delta}_{i h(\tau)} \mathbf{e}_{j}$ - by the $h$-steps-ahead forecast error variance of the $j$ th variable in $\boldsymbol{y}_{t}$, we obtain:

$$
\begin{aligned}
F E V D\left(y_{j t} ; u_{i t(\tau)}, h\right)= & \frac{\omega_{i i,(\tau)}^{-1} \sum_{\ell=0}^{h} \mathbf{e}_{j}^{\prime}\left(\boldsymbol{B}_{\ell(\tau)} \boldsymbol{\Omega}_{(\tau)} \mathbf{e}_{i} \mathbf{e}_{i}^{\prime} \boldsymbol{\Omega}_{(\tau)} \boldsymbol{B}_{\ell(\tau)}^{\prime}\right) \mathbf{e}_{j}}{\sum_{\ell=0}^{h} \mathbf{e}_{j}^{\prime} \boldsymbol{B}_{\ell(\tau)} \boldsymbol{\Omega}_{(\tau)} \boldsymbol{B}_{\ell(\tau)}^{\prime} \mathbf{e}_{j}} \\
& =\frac{\omega_{i i,(\tau)}^{-1} \sum_{\ell=0}^{h}\left(\mathbf{e}_{j}^{\prime} \boldsymbol{B}_{\ell(\tau)} \boldsymbol{\Omega}_{(\tau)} \mathbf{e}_{i}\right)^{2}}{\sum_{\ell=0}^{h} \mathbf{e}_{j}^{\prime} \boldsymbol{B}_{\ell(\tau)} \boldsymbol{\Omega}_{(\tau)} \boldsymbol{B}_{\ell(\tau)}^{\prime} \mathbf{e}_{j}}
\end{aligned}
$$

for $\ell=0,1, \ldots, h$ and $i, j=1, \ldots, m$, where $\mathbf{e}_{j}$ selects the predicted variable and $\mathbf{e}_{i}$ selects the source innovation. Consequently, the generalized forecast error variance decomposition FEVD $\left(y_{j t} ; u_{i t(\tau)}, h\right)$ measures the proportion of the $h$-steps-ahead forecast error variance of the $j$ th variable in $\boldsymbol{y}_{t}$ accounted for by the $i$ th idiosyncratic innovation, $v_{i t(\tau)}$.

\subsection{The Diebold-Yilmaz Approach to Network Analysis}

Based on our definition of the quantile FEVD in (17), it is straightforward to generalize the Diebold-Yilmaz framework for conditional mean network analysis to the conditional quantile setting. The $h$-steps-ahead $m \times m$ spillover matrix for $\boldsymbol{y}_{t}$ evaluated at the $\tau$ th conditional quantile may be written as follows:

$$
\mathbb{A}_{(\tau)}^{(h)}=\left[\begin{array}{cccc}
\theta_{1 \leftarrow 1,(\tau)}^{(h)} & \theta_{1 \leftarrow 2,(\tau)}^{(h)} & \cdots & \theta_{1 \leftarrow m,(\tau)}^{(h)} \\
\theta_{2 \leftarrow 1,(\tau)}^{(h)} & \theta_{2 \leftarrow 2,(\tau)}^{(h)} & \cdots & \theta_{2 \leftarrow m,(\tau)}^{(h)} \\
\vdots & \vdots & \ddots & \vdots \\
\theta_{m \leftarrow 1,(\tau)}^{(h)} & \theta_{m \leftarrow 2,(\tau)}^{(h)} & \cdots & \theta_{m \leftarrow m,(\tau)}^{(h)}
\end{array}\right]
$$


where we apply the row-sum normalization developed by Diebold and Yilmaz (2014) and define $\theta_{j \leftarrow i,(\tau)}^{(h)}=F E V D\left(y_{j t} ; u_{i t(\tau)}, h\right) / \sum_{i=1}^{m} F E V D\left(y_{j t} ; u_{i t(\tau)}, h\right)$ to ensure that each row of $\mathbb{A}_{(\tau)}^{(h)}$ sums to 1 even in the presence of residual cross-correlation. $\theta_{j \leftarrow i,(\tau)}^{(h)}$ measures the spillover of idiosyncratic shocks affecting variable $i$ onto variable $j$. Based on $\mathbb{A}_{(\tau)}^{(h)}$, we may define the following summary measures of the network topology at the $\tau$ th conditional quantile:

$$
\begin{gathered}
O_{i \leftarrow i,(\tau)}^{(h)}=\theta_{i \leftarrow i,(\tau)}^{(h)} \\
F_{i \leftarrow \bullet,(\tau)}^{(h)}=\sum_{\substack{j=1, j \neq i \\
m}}^{m} \theta_{i \leftarrow j,(\tau)}^{(h)} \\
T_{\bullet \leftarrow i,(\tau)}^{(h)}=\sum_{j=1, j \neq i}^{m} \theta_{j \leftarrow i,(\tau)}^{(h)} \\
N_{i \leftarrow i,(\tau)}^{(h)}=T_{\bullet \leftarrow i,(\tau)}^{(h)}-F_{i \leftarrow \bullet,(\tau)}^{(h)} .
\end{gathered}
$$

The proportion of the $h$-steps-ahead FEV of the $i$-th variable that can be attributed to shocks to variable $i$ itself is known as the own variance share, $O_{i \leftarrow i,(\tau)}^{(h)}$. The from (or in) degree of variable $i, F_{i \leftarrow \bullet,(\tau)}^{(h)}$, measures the total spillover from the system to variable $i$. As such, it measures the dependence of variable $i$ on external conditions. Likewise, the to (or out) degree of variable $i$, $T_{\bullet \leftarrow i,(\tau)}^{(h)}$, captures the total spillover from variable $i$ to the system, which measures the influence of the $i$ th node in the network. $N_{i \leftarrow i,(\tau)}^{(h)}$ is therefore a natural measure of the net directional connectedness of variable $i$. Note that $O_{i \leftarrow i,(\tau)}^{(h)}+F_{i \leftarrow \bullet,(\tau)}^{(h)}=1, i=1,2, \ldots, m$ by construction but that $T_{\bullet \leftarrow i,(\tau)}^{(h)}$ can be greater than or less than one. Finally, the spillover index evaluated at the $\tau$ th conditional quantile is given by:

$$
S_{(\tau)}^{(h)}=m^{-1} \sum_{i=1}^{m} F_{i \leftarrow \bullet,(\tau)}^{(h)} .
$$

\section{Credit Spread Data}

Our model includes the following $N=17$ countries: Austria ${ }^{\dagger}$, Australia, Belgium ${ }^{\dagger}$, China, France $^{\dagger}$, Germany ${ }^{\dagger}$, Ireland ${ }^{\dagger}$, Italy ${ }^{\dagger}$, Japan, the Netherlands ${ }^{\dagger}$, Norway* $^{*}$, Portugal ${ }^{\dagger}$, Russia, Spain $^{\dagger}$, Sweden*, the UK* and the US. Eurozone members are marked with a dagger symbol, while European countries that do not use the Euro are marked with an asterisk. Our dataset is sampled at daily frequency over the period 03-Jan-2006 to 29-Dec-2017. For each country, we include two country-specific credit spreads, one for the sovereign and one for the financial 
sector. Unfortunately, despite the fact that it suffered a severe debt crisis during our sample period that raised concerns over the contagion of sovereign credit risk, we are unable to include Greece in our sample, because there is a period of more than a year in 2012-3 where data on the Greek sovereign CDS spread is unavailable. ${ }^{7}$ However, given the severity of the Greek debt crisis and its potential to generate contagion, we re-estimate an enlarged version of our model including Greece over a shorter sample, ending on February 14, 2012. Appendix A contains a concise overview of the results of this exercise and demonstrates that our key findings are not unduly influenced by the forced omission of Greece.

\subsection{Sovereign Credit Risk}

We measure the change in sovereign credit risk using the first difference of the five-year sovereign CDS spread, expressed in basis points. Following the CDS market conventions outlined by Bai and Wei (2017), we work with US dollar denominated CDS in all cases except for the US, where we employ Euro denominated CDS. In addition, we use CDS contracts with a complete restructuring clause for every sovereign except Australia, where we use CDS contracts with a modified restructuring clause as the data is more complete.

\subsection{Financial Sector Credit Risk}

We track changes in financial sector credit risk in the $i$ th country using the first difference of the synthetic sector-wide CDS spreads constructed by Greenwood-Nimmo et al. (2019), which we extend from their original end point of 27-Jul-2015 until 29-Dec-2017. Taking inspiration from the approach of Acharya et al. (2014), Greenwood-Nimmo et al. (2019) construct a synthetic credit spread for the $i$ th country as an equally-weighted average of the CDS spreads for firms that satisfy a variety of selection criteria. Among these criteria, firms must: (i) have USD denominated five-year CDS spread data in the Markit database that conforms to the corporate CDS market conventions documented by Bai and Wei (2017); (ii) be classified by Markit as financials; (iii) be classified as either banking or insurance firms in Bureau van Dijk's Osiris database; (iv) be identified by Markit as operating in the ith country; and (v) hold assets of USD10bn or more. The large majority of the firms included in the indices are publicly traded, although there are two notable exceptions: (i) in Austria, Raiffeisen Zentralbank is included in

\footnotetext{
${ }^{7}$ This gap in the data reflects the fact that Greek sovereign CDS contracts ceased trading on a running spread in favor of a points upfront convention in 2012 due to the expectation of an imminent credit event. In such an environment, protection sellers benefit from a points upfront arrangement, because it eliminates the possibility that they will have to pay out before they have received any income from the contract.
} 
the sample, to ensure that the index is not based on data for a single firm; and (ii) in China, data for four large state-sponsored banks is used, as there is not enough CDS data for privately held Chinese banks to construct a meaningful index. Similarly, rather than simply dropping failed banks from the sample, Greenwood-Nimmo et al. (2019) include CDS data for several institutions that became state-owned as a result of the crisis, such as the Irish Bank Resolution Corporation. ${ }^{8}$

The financial sector CDS spreads that we use are derived from data on 199 financial institutions, a sample that provides good coverage of the global financial system. A full list of the financial institutions included in the synthetic financial sector credit spread for each country may be found in Appendix C. This list includes 28 of the 30 Globally Systemically Important Banks (G-SIBs) and 8 of the 9 Globally Systemically Important Insurers (G-SIIs) identified by the Financial Stability Board as of November $2017^{9}$. Of the three omitted G-SIBs and G-SIIs, one is domiciled in Canada and so lies is outside of the scope of our analysis, while the remaining two (China Construction Bank and Ping An Insurance Group Ltd.) are omitted due to a lack of data. Our list also includes the majority of Domestically Systemically Important Banks (D-SIBs) domiciled in our sample countries and offers comparable cross-sectional coverage to leading empirical studies of the connectedness of the financial system (e.g Demirer et al., 2018).

\subsection{Properties of the Dataset}

Table 1 provides elementary summary statistics for the dataset. Preliminary analysis of the autocorrelation structure in the data indicates that each series is stationary with relatively limited serial correlation (results are available on request). The countries in our sample form two natural groups, one composed of Ireland, Italy, Portugal, Russia and Spain, all of which display high and volatile credit spreads, and the other composed of the remaining countries in

\footnotetext{
${ }^{8}$ For a detailed discussion of the construction and properties of the financial sector CDS spreads, the reader is referred to Greenwood-Nimmo et al. (2019) and, in particular, to their Data Supplement. To ensure that our financial sector credit spreads are identical to those developed by Greenwood-Nimmo et al. (2019) up to the point at which their sample ends (27-Jul-2015), we retain their original selection criteria precisely. To construct data beyond this point, we take the list of financial institutions for each country as of 27-Jul-2015 as given and construct the financial sector credit spread for the remainder of the sample as a simple equally-weighted average. The use of an equally-weighted average of single name CDS spreads as opposed to an asset-weighted average, for example, is consistent with the seminal study of Acharya et al. (2014). Furthermore, Greenwood-Nimmo et al. (2019) note that the choice of weighting scheme does not exert a strong influence over the resulting index due to the strong positive correlation among the CDS spreads for the set of financial institutions in each country. In fact, in their sample, they report that the correlation between the equally-weighted financial sector credit spreads and an alternative definition based on fixed asset weights is approximately 0.97 on average across countries.

${ }^{9}$ See https://www.fsb.org/wp-content/uploads/P211117-1.pdf for the list of G-SIBs and https://www . fsb.org/wp-content/uploads/2016-list-of-global-systemically-important-insurers-G-SIIs.pdf for the list of G-SIIs. Note that the Financial Stability Board did not update the G-SII list in 2017, so the 2016 list remained current at the end of our sample.
} 
our sample, which display considerably lower and less volatile credit spreads. For any given country, the sovereign CDS spread is typically lower and less volatile than the financial sector CDS spread. In general, sovereign credit spreads should act as a lower bound on financial sector credit spreads, not least because of the implicit guarantee that the sovereign extends to the financial sector. However, due to their deep sovereign crises, this is not the case in Italy or Spain.

— Insert Table 1 here -

The credit spreads for all countries display pronounced excess kurtosis, reflecting the severity of the shocks affecting global financial markets over our sample period. The evidence of heavy tails in the data suggests that estimation by quantile regression may be preferable to the use of simple conditional mean estimators, not just because it can illuminate tail relationships, but also because it is more robust in the presence of extreme observations.

\section{Estimation Results}

Before we proceed, we must first determine appropriate values for both the QFVAR lag order and the forecast horizon used in the construction of our connectedness measures. We follow the precedent of Koenker and Xiao (2006) and set the lag order at every conditional quantile equal to the optimal lag order that is selected at the conditional mean by minimization of the Schwarz Information Criterion. This results in a first-order model, which we verify is dynamically stable - the largest eigenvalue of the companion matrix is just $0.34 .{ }^{10}$ Unfortunately, there is no similar rule to select an optimal forecast horizon for connectedness analysis. For this reason, we compare the properties of the adjacency matrix evaluated at the conditional mean for three different forecast horizons, $h \in\{3,5,10\}$ trading days. We select relatively short horizons in light of the fact that the FEVDs obtained from a first order VAR model estimated on data with a low degree of persistence are likely to rapidly converge to their long-run values. This observation is borne out by our finding that the network statistics are largely invariant to the choice of horizon within this range, with the spillover indices obtained under $h=3, h=5$ and $h=10$ being identical to the first decimal place (an elementwise comparison of the spillover matrices is available on request). A similar degree of invariance with respect to the forecast

\footnotetext{
${ }^{10}$ In practice, allowing for higher lag orders does not change our results substantially - the distributions of the pairwise spillover effects obtained from the factor $\operatorname{VAR}(1), \operatorname{VAR}(2)$ and $\operatorname{VAR}(3)$ models are all very similar. Further details are available from the authors on request.
} 
horizon has been documented by Greenwood-Nimmo et al. (2016) in the context of a stationary first order VAR model applied to the analysis of risk spillovers among the G10 currencies. We therefore adopt a forecast horizon of five trading days without loss of generality, although we shall return to the issue of horizon selection in the rolling sample context in Section 4.3.

\subsection{Interpretation of the Factors}

We allow for up to 15 common factors at the conditional mean and at each conditional quantile. Moreover, to achieve the greatest possible flexibility, we allow the number of factors to vary between quantiles, with the optimal number being selected in each case by minimization of the information criterion introduced by Ando and Bai (2020). The number of factors chosen in each case is reported in Figure 1. At the conditional mean, ten factors are selected. Interestingly, there are only 9/99 conditional quantiles where more factors are selected and they all lie far into the far left and right tails of the conditional distribution. In the center of the conditional distribution, either five or six common factors are typically selected. This suggests that more sources of common influence are relevant when large shocks affect the system than when smaller shocks prevail.

— Insert Figure 1 here -

In practice, a finite number of common factors is unlikely to completely eliminate the crosssection correlation among the regression residuals but it should attenuate it sufficiently to allow us to proceed on the basis of approximate cross-section independence. The adequacy of our factor structure is ultimately an empirical question. To evaluate the degree to which our factor structure is able to account for the cross-section correlation among the residuals, it is necessary to compare two models, one with a factor structure and one without. This would not be a straightforward undertaking for the QFVAR model, as the presence of non-trivial cross-section correlations among the regression residuals would frustrate efforts to properly define the conditional quantiles. Therefore, we limit our attention to the conditional mean case and compare the residual cross-correlations observed from a simple VAR(1) model with no factors and from our factor VAR(1) model. Figure 2(a) reveals considerable correlation among the residuals of the simple $\operatorname{VAR}(1)$ model, with more than half of the pairwise correlation coefficients exceeding 0.25 in absolute value and almost one-quarter exceeding 0.4. Figure 2(b) shows that the factor structure removes a great deal of this correlation. In the factor $\operatorname{VAR}(1)$ 
model, $91.6 \%$ of the pairwise correlations are weaker than 0.25 in absolute value and $97.9 \%$ are weaker than 0.4. This strongly supports the validity of our factors and suggests that it is a reasonable simplification to treat the error terms of the factor VAR(1) model as cross-sectionally uncorrelated. $^{11}$

\section{— Insert Figure 2 here -}

The factor loadings (available on request) indicate that the unobserved common factors capture three main phenomena. First, there are factors that load on all credit spreads to varying degrees and that capture global variations in aggregate credit risk. Next, there are factors that load heavily on either financial sector or sovereign credit spreads. These factors appear to capture financial-sector-specific and sovereign-specific credit risk. Finally, there are factors that capture the impact of sovereign instability. For example, several of the factors load heavily on subsets of the sovereign and financial sector credit risk measures for Ireland, Italy, Portugal, Spain and Russia. These are the countries in our sample that have experienced the most profound sovereign instability and that have raised the greatest concerns over sovereign credit risk contagion.

It is natural to ask whether the unobserved common factors that we obtain are related to any observable macroeconomic or financial variables. To address this question, we compute the correlation between the unobserved factors that we obtain at the conditional mean and median and at the 5th and 95th conditional quantiles against a list of observed macroeconomic and financial variables that is inspired by those considered by Longstaff et al. (2011) in their analysis of the common components in sovereign credit spreads. First, we use the three Fama-French factors and the 5-year US constant maturity treasury (CMT) yield to proxy for global economic and financial conditions. Next, we use the TED spread, the Euribor-DeTBill spread, the US equity variance risk premium, the 5-year US Treasury term premium and the US investment grade (IG) and high yield (HY) bond spreads to capture variations in funding liquidity and investor risk appetite. We also include an array of five iTraxx credit risk indices to capture

\footnotetext{
${ }^{11}$ The cross-section dependence (CD) test statistic of Pesaran $(2004,2015)$ is increasingly being applied to the residuals of regression models for use as an ex-post diagnostic tool. In practice, the residual-based CD test has been shown to often reject the null hypothesis of no remaining cross-section dependence in the case of the Common Correlated Effects (CCE) estimator (e.g. Mastromarco, Serlenga and Shin, 2016). Juodis and Reese (2018) show that the application of the CD test to regression residuals obtained from two-way fixed effects models or interactive effects models introduces a bias term of order $\sqrt{T}$, which may lead to an erroneous rejection of the null hypothesis. Nonetheless, the CD test may be used as a model-selection tool, with a reduction in the absolute value of the CD test statistic typically being interpreted as an indication of an improved model specification. Used in this manner, the reduction in the value of the CD test statistic from 393.09 for the VAR(1) model with no factors to 9.924 for our factor VAR(1) model suggests that our factor structure leads to a substantial improvement in the specification of the model.
} 
pan-European credit risk factors, as well as the daily log-return of the bilateral spot exchange rate for each currency in our sample, in units of foreign currency per USD. Further details of the construction of these variables and the relevant data sources may be found in Appendix D.

Figure 3 reveals that the strongest correlations between the unobserved factors obtained at all conditional quantiles and the list of observed global variables arises in the case of the iTraxx credit risk indices. This is perhaps unsurprising, as the iTraxx indices are designed to capture different aspects of aggregate credit risk. Several of the unobserved factors are also correlated with the market excess return and the equity variance risk premium, an observation that accords with the results of Longstaff et al. (2011). However, it is important to note that several of the unobserved factors are essentially uncorrelated with any of the global variables that we identify. This highlights the value of using an unobserved factor approach, because these sources of common variation do not appear to exhibit any obvious relationship to observable global macroeconomic or financial conditions. Consequently, it may not be possible to capture these factors in an observed factor framework.

— Insert Figure 3 here -

\subsection{Conditional Mean vs. Conditional Quantiles}

To establish a point of reference for the analysis that follows, we begin by comparing the spillover index obtained from the model evaluated at the conditional mean with the values obtained from the model evaluated at conditional quantiles $\tau=0.1,0.2, \ldots, 0.99$. This is an important exercise, because if the results from network models evaluated at the conditional mean are to be generalized across the conditional distribution — as is currently the norm in the network literature, at least implicitly — then the results obtained at the conditional mean and at each conditional quantile should be similar. However, there is reason to believe that the results will differ non-trivially given the extreme credit spread movements recorded in several countries during our sample. Such extreme observations can be naturally accommodated by quantile regression but may compromise the reliability of a conditional mean estimator.

Our interpretation of the quantile regression estimates will be predicated on the distinction between large adverse shocks and large beneficial shocks. To see this, note that in the right tail of the conditional distribution, the observed changes in the vector of credit spreads are large conditional on the values taken by the explanatory variables - that is, credit spreads are increasing sharply due to the effect of large adverse shocks. By contrast, in the left tail, credit 
spreads are falling sharply conditional on the explanatory variables, due to the impact of large beneficial shocks.

In light of the evidence that financial market comovements increase under adverse conditions (e.g. Ang and Bekaert, 2002), it is natural to think that strong spillovers should be observed in the right tail of the conditional distribution, where the largest adverse shocks affect the system. Figure 4 reveals this to be the case: the spillover intensity in the upper quantiles is approximately twice as strong as at the conditional median. ${ }^{12}$ Interestingly, however, the figure also reveals that strong spillovers occur in the left tail of the conditional distribution, indicating that spillover intensity increases with shock size for both adverse (right tail) and beneficial (left tail) shocks. Over the central $40 \%$ of the conditional distribution, the spillover index fluctuates at around $40 \%$, indicating that the spillover of idiosyncratic credit risk shocks is dominated by the effect of variable-specific shocks when small shocks affect the system. By comparison, when large idiosyncratic shocks affect the system, bilateral spillovers play a profound role in shaping the evolution of sovereign and financial sector credit risk. At the 1st, 5th, 10th, 90th, 95th and 99th conditional quantiles, the spillover index takes values of $88.18 \%, 77.17 \%, 72.20 \%, 73.34 \%$, $79.36 \%$ and $91.77 \%$, respectively. This profound increase in the spillover intensity in both tails of the conditional distribution is consistent with the literature on good and bad contagion, which emphasizes the transmission of unexpected events in both the left and right tail (e.g. Jorion and Zhang, 2007; Londono, 2019).

\section{— Insert Figure 4 here -}

The large tail effects reported in Figure 4 are not simply due to a lack of effective observations in the tails of the conditional distribution. For any given $\tau$, quantile regression makes use of every data point with non-zero weight and our sample of 3,129 trading days is sizable compared to the dimensionality of the QFVAR system. Rather, our results are consistent with the hypothesis that the informational content of large shocks is greater than that of small shocks, a point that is well-established in the volatility literature (e.g. Dendramis, Kapetanios and Tzavalis, 2015). When a large idiosyncratic shock affects a given sovereign or financial sector, investors gain significant news that may lead to a reappraisal of the level of risk associated with other nodes in the system. Consequently, the quantile variation documented in Figure 4 can be interpreted like a regime-switching process, in which the transition between regimes of strongly beneficial

\footnotetext{
${ }^{12}$ See Figure A1 in Appendix A for a replication of Figure 4 based on the model including Greece estimated on a sample ending in February 2012. The similarity between the two figures indicates that our results are robust to the inclusion of Greece.
} 
news at one extreme and strongly adverse news at the other occurs relatively smoothly as the shock size varies. It is interesting to note that the pattern of quantile-variation in the spillover index is approximately symmetric over the full sample. However, this near-symmetry is not a general feature of our results, because we will demonstrate below that it breaks down in rolling sample analysis. We will return to this issue shortly.

To provide an impression of how the differences in aggregate spillover intensity surveyed above are reflected in the network topology, Figures 5 and 6 provide schematic visualizations of the network evaluated at the conditional mean and median and at the fifth and ninety-fifth conditional quantiles. Sovereigns are represented by white nodes and financial sectors by shaded nodes, while the strength of bilateral spillovers is indicated by the relative thickness of the edges. The layout of all four graphs is identical and is determined using the force-directed algorithm of Fruchterman and Reingold (1991) applied at the conditional mean.

\section{- Insert Figures 5 \& 6 here -}

The network visualizations display some similar features, notably the centrality of the crisis-hit peripheral European sovereigns and the disposition of many of the strongest bilateral spillover effects. However, some important differences are easily seen, most notably that, although the average bilateral spillover effect is stronger in the tails than at the conditional mean or median (as reflected in the spillover index reported in Figure 4), the strongest individual pairwise spillover effects are actually observed at the conditional mean and at the center of the conditional distribution. A similar finding is documented by Dungey, Harvey and Volkov (2019), who demonstrate that a smaller number of strong bilateral linkages may be replaced by a greater number of weaker bilateral linkages during times of stress, resulting in an increase in the weighted completeness of the sovereign-financial credit risk network.

This feature of the network is most clearly demonstrated by inspection of the distribution of bilateral spillover effects (henceforth the spillover density) at the conditional mean and at selected quantiles. For convenience, we multiply each element of the adjacency matrix by 100, so that the estimated spillover effects can be interpreted as percentages rather than proportions. In principle, therefore, the spillover density has support $[0,100]$ although, in practice, the limiting cases of 0 and 100 will only arise from restricted VAR models in which the parameter and covariance matrices are sparse. Note that the spillover density of a network constructed by the Diebold-Yilmaz method resembles a power law. ${ }^{13}$ This is a natural finding given that

\footnotetext{
${ }^{13}$ Power law behavior is pervasive in economic and financial networks - see Acemoglu, Carvalho, Ozdaglar
} 
the elements of the adjacency matrix are constructed from variance decompositions, which are defined as ratios of quadratic forms. Consequently, for ease of interpretation, we report empirical counter cumulative distribution functions (CCDFs) on a logarithmic scale in Figure 7.

\section{— Insert Figure 7 here -}

Figures 7(a) and 7(b) plot the spillover density at the conditional mean and median. Several features are noteworthy. First, the right tails of both densities are similar, which indicates that the strongest spillovers in the system are of comparable magnitude at the conditional mean and median. The three strongest bilateral spillovers at the conditional mean are from the Belgian financial sector to the Dutch financial sector $(24.22 \%)$ and from the Russian financial sector to the US and Spanish financial sectors, respectively (17.37\% and 16.94\%). By contrast, at the conditional median, the three strongest pairwise spillovers are from the Spanish sovereign to the Italian sovereign $(22.75 \%)$, from the Italian sovereign to the Spanish sovereign $(22.29 \%)$ and from the Chinese sovereign to the Chinese financial sector $(16.79 \%)$. The evidence of strong spillovers involving the European periphery (including Belgium, which narrowly avoided a sovereign debt crisis in 2011) reflects their central role in the European debt crisis.

The similarity in the right tail of the spillover density does not extend to other parts of the density, however. The CCDF is less curved and displays considerably greater left tail mass at the conditional median than at the conditional mean, implying a higher proportion of weak spillovers at the median than at the mean. To appreciate the difference in the shape of the two spillover densities more clearly, consider an arbitrary threshold — of the 1,122 bilateral spillovers that we study, 568 are weaker than $1 \%$ at the conditional mean but this value rises to 829 at the conditional median.

The shift toward a greater number of weaker spillovers in both tails of the conditional distribution can be seen clearly in Figures 7(c)-(f). Note how the spillover density becomes more peaked and the left and right tails both shrink as $\tau \rightarrow 1$ and $\tau \rightarrow 0$. This results in a substantial increase in the average intensity of bilateral spillovers, implying that large idiosyncratic credit risk shocks propagate considerably more widely than weaker shocks.

The granular differences across quantiles documented above accumulate substantially under aggregation. Table 2 reports the to (weighted out-degree), from (weighted in-degree) and net statistics for each node in the system at the conditional mean and at the fifth and ninety-fifth conditional quantiles. In the large majority of cases, the conditional mean estimate of the and Tahbaz-Salehi (2012) and Gabaix (2016), for example. 
weighted in- and out-degrees is smaller than the estimates obtained in either the left or the right tails, often substantially so. Exceptions to this general rule occur mainly in the sovereigns and financial sectors that experienced the most profound crises during our sample. The large shocks experienced by each of these nodes may explain the high weight that is attached to them under conditional mean estimation.

— Insert Table 2 here -

A natural question to ask at this stage is whether the ranking of nodes by centrality is preserved across quantiles. That is, does the group of nodes that display the strongest outward spillovers vary with the shock size? To this end, in Figure 8, we plot the weighted out-degree rank of each node in the system, with the nodes ranked in decreasing order of influence. The weighted out-degree captures the total strength of all outward spillovers originating from a given node. As such, it represents a natural measure of the influence of a node.

— Insert Figure 8 here -

The figure is organized with the peripheral European countries (Ireland, Italy, Portugal and Spain) on the top row, the other European countries on the next two rows and the non-European countries on the final row. Several features of Figure 8 are noteworthy. First, for the majority of European sovereigns - both core and peripheral — the weighted out-degree rank is relatively high across most of the conditional distribution, with the German sovereign consistently ranked close to the top. This reflects the integration of the European sovereign bond market over much of our sample period and the unique role of German bonds as reference points within this market. This also explains why Norway, which is the only European country in our sample that is not a member of the European Union, achieves a relatively low sovereign out-degree rank at most conditional quantiles. Interestingly, the peripheral European sovereigns that experienced pronounced sovereign debt crises do not dominate the network. One plausible explanation of this phenomenon is that the degree of spillover from the European periphery onto other global sovereigns at the height of the sovereign debt crisis was limited by their decoupling from the major global sovereign bond markets.

The Chinese sovereign and financial sector stand apart in our analysis. The Chinese sovereign generates the strongest outward credit risk spillovers of any entity in the system at the center of the conditional distribution and the Chinese financial sector also generates 
strong spillovers in this region. However, a substantial proportion of this spillover activity is bilateral, reflecting the fact that the Chinese financial system is mostly populated by largely or wholly state-sponsored institutions.

The outdegree rank of most sovereigns drops in the extreme left and right tails of the conditional distribution. Two most notable exceptions to this rule are the Japanese and US sovereigns, bonds issued by which are known to serve as safe haven assets in turbulent times. The reason that the out-degree rank of most other sovereigns falls in the tails is that it is the financial sector that plays a dominant role in the propagation of the largest shocks affecting the system. Indeed, the large and potentially fragile French financial sector is among the top sources of credit risk in our model across much of the upper and lower tails.

Figure 8 focuses on the multilateral spillover effects originating from each node in the system. It does not convey information about the bilateral relationship between the credit risk of the $i$ th sovereign and the $i$ th financial sector, which has received a great deal of attention in the recent literature. It is these bilateral interactions that are central to the feedback loops described by Acharya et al. (2014) and Farhi and Tirole (2018). In our model, the feedback between the $i$ th sovereign and the $i$ th financial sector is captured by the sum of the bilateral spillovers between the two nodes, $\mathcal{T}_{s_{i} \leftrightarrow f_{i},(\tau)}^{(5)}=T_{s_{i} \leftarrow f_{i},(\tau)}^{(5)}+T_{f_{i} \leftarrow s_{i},(\tau)}^{(5)}$. Figure 9 reports the quantile variation in $\mathcal{T}_{s_{i} \leftrightarrow f_{i},(\tau)}^{(5)}$ for all 17 countries in our sample.

\section{— Insert Figure 9 here —}

Setting aside the special case of China, where the distinction between financial sector and sovereign credit risk is obscured by state involvement in the financial sector, the feedback effect is typically negligible throughout the center of the conditional distribution. However, the feedback intensifies markedly in both tails, particularly in the European periphery and in Russia. This is potentially an important result, because the adverse feedback loops described by Acharya et al. (2014) and Farhi and Tirole (2018) focus on the feedback associated with adverse shocks, in the right tail of the conditional distribution. Our results indicate that a virtuous feedback loop may also act upon the arrival of large beneficial shocks. We conjecture that, in some cases, a sovereign bailout may offer an example of such a beneficial shock. Suppose that the $i$ th sovereign receives a bailout, which reduces its credit risk. This increases the ability of the $i$ th sovereign to stabilize the $i$ th financial sector, while simultaneously lowering the default risk associated with domestic sovereign bonds held by the $i$ th financial sector. This leads to a reduction in financial sector credit risk, which lowers the probability that the sovereign will be required to 
intervene in the financial sector, further lowering sovereign risk and so on. This suggests that the same feedback loop that promotes instability in the analysis of Acharya et al. (2014) can act to restore stability if policymakers are able to generate large beneficial shocks.

\subsection{Time-Varying Tail-Dependence}

To this point, our analysis has focused exclusively on full-sample statistics. We have demonstrated substantial quantile-variation in the topology of the credit risk network and have shown that bilateral spillovers of idiosyncratic credit risk are considerably stronger in the tails than they are at the conditional median. This implies that network models estimated at the conditional mean are unlikely to adequately capture the full extent of dependence when large shocks occur. As noted by Betz et al. (2016), it is tail-dependence that should be of the greatest interest for surveillance and regulatory purposes. Unlike the existing literature on Diebold-Yilmaz networks, by estimating our QFVAR model on a rolling sample basis, we can directly study time-variation not only in average connectedness but also in the extent of left- and right-taildependence.

Before we proceed, we must first choose an appropriate window length for our rolling samples. Existing studies in the Diebold-Yilmaz network literature that work with daily data have used a variety of window lengths, including 100 days (e.g. Diebold and Yilmaz, 2014), 150 days (e.g. Demirer et al., 2018), 200 days (e.g. Baruník et al., 2016) and 250 days (e.g. Greenwood-Nimmo et al., 2016, 2019). In the absence of a firm precedent, we evaluate the sensitivity of our results to a set of three candidate window lengths, $w \in\{250,375,500\}$ trading days. We do not consider shorter windows to ensure that we do not encounter small-sample issues in estimation. Small sample problems are always a concern in rolling regression exercises and may be particularly problematic in the context of quantile regressions, where they may lead to a lack of effective observations in the tails of the conditional distribution. In addition, we take this opportunity to further explore the robustness of our results to the choice of forecast horizon, $h \in\{3,5,10\}$.

Either of two approaches could be used to determine the relevant number of common factors in each rolling sample. First, one could select the optimal number of common factors in each rolling sample using the Ando and Bai (2020) procedure. In principle, this approach accounts for the possibility that the number of relevant factors may differ between periods of turbulence and periods of tranquility. However, as a practical matter, it may be difficult to precisely estimate the number of common factors in each rolling sample using just 250 observations and 
this approach may result in variations in the number of common factors from one rolling sample to the next that are difficult to reconcile. Second, one could set the number of common factors in each rolling sample equal to the number selected over the full sample. This approach is simpler, computationally cheaper and has the significant benefit that it may facilitate comparisons across rolling samples, because any observed time-variation in the structure of the network cannot be attributed to changes in the number of factors. This is the approach that we adopt. We evaluate the sensitivity of our results to this choice in Appendix B, where we compare a selection of key rolling sample network statistics obtained under both procedures. Our results prove highly robust, a finding that supports adoption of the simpler option.

Figure 10 reports the spillover index under the nine possible combinations of window length and forecast horizon at the conditional mean and median, as well as the fifth and ninety-fifth conditional quantiles. First, consider panels (a) and (b). At the conditional mean and median, the choice of forecast horizon has little discernible effect on the spillover index, a result that reinforces our findings over the full sample. The choice of window length affects the level of the spillover index, with shorter windows yielding somewhat higher values. Nonetheless, it is the dynamics of the spillover index that are of primary concern and they are similar irrespective of the choice of window length. The correlation among the spillover indices obtained under the nine different combinations of $w$ and $h$ is close to one in all cases at the conditional mean and is somewhat lower but still strongly positive in all cases at the conditional median. The same basic features are also apparent at the fifth and ninety-fifth conditional quantiles, although with greater noise. Critically, however, the correlation across different combinations of window length and forecast horizon remains strongly positive in the tails. We therefore conclude that the choice of window length and forecast horizon does not exert an undue influence on our results. Therefore, we proceed with $w=250$ and $h=5$ trading days without loss of generality, resulting in a total of 2,880 rolling samples. The benefit of choosing the shortest window length is that fewer observations are required for initialization, allowing our rolling sample analysis to start at an earlier date.

— Insert Figure 10 here -

With the forecast horizon and window length set, Figure 11(a) re-plots the spillover index evaluated at the conditional mean and median — both of which are different measures of the average connectedness of the system - on the same axes. To assist the reader, the dates of several important events are marked by vertical dashed lines. Figure 11(b) plots the spillover 
index evaluated at the fifth conditional quantile as a measure of left-tail-dependence. This captures the propensity for beneficial shocks that reduce credit risk to propagate through the system. All else equal, stronger left-tail-dependence is stabilizing. Figure 11(c) plots the the spillover index at the ninety-fifth conditional quantile as a measure of right-tail-dependence, the propensity for destabilizing adverse shocks to spread. Lastly, Figure 11(d) plots the linear combination $R T D=S_{0.95}^{(5)}-S_{0.05}^{(5)} \cdot{ }^{14} R T D$ has a natural interpretation as a measure of relative tail-dependence, with positive (negative) values indicating stronger (weaker) dependence in the right tail than in the left tail. We therefore interpret increases (decreases) in $R T D$ as evidence of rising (falling) financial fragility. The correlations between the the four different spillover indices and our RTD measure are reported in Table 3.

\section{— Insert Figure $11 \&$ Table 3 here -}

The time-variation that we observe in the $R T D$ implies that the near-symmetry of Figure 4 in the full-sample setting is not preserved over rolling samples. To see this, note that the full sample $R T D$ takes a value of just $2.19 \%$ due to the near-symmetry of the spillover index across the conditional distribution (it would be precisely zero if Figure 4 was exactly symmetric). The fact that we observe values of the order of $\pm 15 \%$ in the rolling sample $R T D$ indicates that the spillover index is strongly asymmetric across the conditional distribution at certain points in time. Our conjecture is that the $R T D$ may rise significantly above zero during adverse episodes of elevated financial fragility, perhaps due to the short-run failure of mechanisms that typically act to stabilize the financial system (e.g. arbitrage opportunities may not be fully exploited in bad states of the world due to factors including liquidity constraints). Likewise, RTD may fall below zero during beneficial episodes, when risks are rapidly receding (e.g. in the wake of large-scale policy interventions intended to support financial stability). Over a long sample that combines several adverse and beneficial episodes, the $R T D$ may tend toward zero.

The spillover indices evaluated at the conditional mean and median display broadly similar behavior and Table 3 reveals that they are strongly positively correlated (0.72). Both increase abruptly as a result of major adverse shocks, such as the freezing of redemptions in selected investment funds by BNP Paribas in August 2007 and the bankruptcy of Lehman Brothers in September 2008. Similarly, beneficial shocks, such as the announcement of the Troubled Asset

\footnotetext{
${ }^{14}$ See Figure A2 in Appendix A for a replication of Figure 11 based on an enlarged model including Greece estimated using data up to February 2012. The dynamics of the spillover indices and the $R T D$ obtained from both models are remarkably similar over the common sample period, indicating that our results are robust to the inclusion of Greece. Likewise, Figures B1 and B2 in Appendix B reveal that our assumption that the number of common factors is unchanged across rolling samples does not strongly influence the results.
} 
Relief Program (TARP), the American Recovery and Reinvestment Act (ARRA), several of the European sovereign bailouts and the announcement of negative interest rate policy by the European Central Bank (ECB), are all associated with reductions in both indices.

A natural question is whether the spillover indices evaluated at the conditional mean and median share common dynamics with the spillover indices evaluated in the tails of the conditional distribution. The correlations in Table 3 provide an interesting answer. Both are strongly positively correlated with our measure of right-tail-dependence ( 0.77 and 0.59 , respectively) but are considerably more weakly correlated with our measure of left-tail-dependence $(0.41$ and 0.33 , respectively). Furthermore, our measures of left- and right-tail-dependence are moderately correlated with one-another (0.37) and often move in opposite directions, at least in the shortto medium-term. It is this last result that is most interesting, as it suggests that changes in right-tail-dependence do not necessarily coincide with similar changes in left-tail-dependence and vice-versa. This distinction between the evolution of spillover activity in either tail of the conditional distribution may arise from the aggregate behavior of market participants if the information revealed by a major event in either tail causes a non-trivial proportion of market participants to focus disproportionately on further events occurring in that tail, while paying less attention to events in the other tail.

The differential dynamics of left- and right-tail-dependence is easily seen in Figures 11(b)(d). Consider the revelation of major losses at the Bear Stearns High-Grade Structured Credit Fund and the Bear Stearns High-Grade Structured Credit Enhanced Leveraged Fund in July 2007 as an example of a large adverse shock that led market participants to re-evaluate the level of risk associated with mortgage-backed securities. At this time, we observe an abrupt and long-lasting increase in the sensitivity of market participants to adverse shocks. By contrast, there is a dip in left-tail dependence at this time, indicating a reduced propensity for spillovers of good news. This combination generates a marked increase in $R T D$.

Now, consider the announcement of TARP as an example of a major beneficial shock. TARP represented a substantial government intervention in the financial markets, providing funds for the purchase of toxic assets and equity from troubled financial institutions. The introduction of TARP is associated with a gradual reduction in right-tail-dependence but with an abrupt increase in left-tail-dependence that is sustained for several months. Likewise, the introduction of negative interest rate policy by the ECB generates an abrupt and long-lasting drop in righttail dependence coupled with a gradual increase in left-tail dependence. Recall that we have 
controlled for a raft of common factors, so these results are not simply a manifestation of improved funding liquidity or enhanced investor risk appetite driven by the enactment of these stabilization policies. Rather, our results suggest that major policy interventions can increase the sensitivity of the market to beneficial shocks relative to adverse shocks.

To rule out the possibility that the behavior of $R T D$ documented above is simply an artifact of our choice to work with the fifth and ninety-fifth conditional quantiles, we plot two alternative measures of relative tail-dependence in Figure 12. Specifically, alongside our benchmark 5\% $R T D$, we plot the $10 \%$ and $1 \% R T D$ s defined as $R T D_{10 \%}=S_{0.90}^{(5)}-S_{0.10}^{(5)}$ and $R T D_{1 \%}=$ $S_{0.99}^{(5)}-S_{0.01}^{(5)}$, respectively. The dynamics of the three $R T D$ measures are similar, indicating that our results are robust to the precise definition of the left- and right-tail-dependence measures.

— Insert Figure 12 here -

$R T D$ represents a novel data-driven measure of aggregate systemic fragility that can be updated in close-to-real-time. As such, $R T D$ can be used by policymakers and practitioners alike to augment their systems for monitoring systemic risk. Unlike many of the existing frameworks for monitoring the contribution to systemic risk of individual entities, $R T D$ provides an indication of the extent of aggregate systemic fragility. Increases in $R T D$ indicate that the financial system is becoming more susceptible to the spread of adverse shocks, while decreases in $R T D$ indicate that the financial system is becoming more robust to adverse shocks. As an aggregate tail risk measure, $R T D$ bears a non-trivial similarity to the CATFIN index proposed by Allen et al. (2012). CATFIN is an aggregate downside risk measure derived from the cross-section of financial stock returns. Specifically, CATFIN is constructed as the equally-weighted average of the 99\% VaR computed using the generalized Pareto distribution, the skewed generalized error distribution and a nonparametric method based on the empirical return distribution. Unlike CATFIN, RTD is a measure of relative tail risk that exploits information from both the upper and lower tails of the conditional distribution.

In Figure 13, we investigate the degree to which the $R T D$ and its constituent parts comove with CATFIN over our sample period. As the CATFIN index is only available at monthly frequency, we construct monthly versions of our indices by taking the period-average. In addition, to facilitate comparisons, all series are normalized by subtracting their respective means and dividing by their standard deviations. CATFIN comoves strongly with the spillover index evaluated at the ninety-fifth conditional quantile, with a correlation of 0.51 . By contrast, there is little correlation between CATFIN and our measure of left-tail-dependence (0.15). This is a 
natural finding, because CATFIN is a downside risk measure, and downside risks are associated with the right tail in our framework. The $R T D$ index tracks CATFIN reasonably closely, with a correlation of 0.42. Furthermore, RTD and CATFIN share a number of common turning points. Given the extent of this comovement and bearing in mind that $R T D$ captures a related but subtly different phenomenon than CATFIN, we argue that the two are complementary and that they jointly provide policymakers and practitioners alike with a rich framework for the study of aggregate changes in systemic risk.

— Insert Figure 13 here -

\section{Concluding Remarks}

In this paper, we develop a new technique for the econometric analysis of financial networks in which the topology of the network is allowed to vary with the size of the shocks that affect the system. Our approach is based on a novel framework for the estimation of vector autoregressions by quantile regression. We employ an unobserved factor structure to isolate the idiosyncratic component of the error process from the systematic component. Not only does this align our model with the long literature on systematic and idiosyncratic risk but it also simplifies the estimation problem, as it accounts for the the cross-section correlation among the regression residuals. This greatly simplifies the task of defining regression quantiles and, as a result, our approach is considerably easier to implement than the competing frameworks for the estimation of quantile VARs associated with Cecchetti and Li (2008) and Schüler (2014).

We apply our technique to study the transmission of credit risk among a panel of 17 sovereigns and their respective financial sectors between January 2006 and December 2017. We document marked quantile variation in the topology of the network. We show that, on average, idiosyncratic credit risk shocks do not propagate strongly at the conditional mean or median but that the average spillover intensity is strong in both tails of the conditional distribution. By studying the bidirectional feedback between each sovereign and its domestic financial sector, we find that the adverse feedback loop between sovereign credit risk and financial sector credit risk documented by Acharya et al. (2014) and Farhi and Tirole (2018), among others, manifests as a marked intensification of feedback in the right tail, where large adverse shocks occur. Interestingly, however, we note a similar intensification in the left tail, which indicates that a comparable virtuous feedback loop can act in a stabilizing manner in the presence of 
large beneficial shocks, such as sovereign bailouts.

We use rolling sample analysis to document time-variation in the degree of tail-dependence. This reveals an interesting phenomenon - the level of left-tail-dependence is not strongly correlated with the level of right-tail-dependence. We exploit this observation to develop a measure of relative tail dependence, $R T D$, which is defined as the difference between the spillover index obtained at the ninety-fifth and fifth conditional quantiles. We show that increases in $R T D$ are associated with large adverse shocks, while many stabilizing policy interventions are associated with reductions in $R T D$. We propose that $R T D$ can be used as an aggregate measure of systemic financial fragility in a similar vein to the CATFIN index proposed by Allen et al. (2012). Because the evolution of RTD is obscured when network models are estimated at the conditional mean by OLS, as is common in the literature, we conclude that our quantile connectedness framework represents a valuable addition to the literature on empirical network modeling. 


\section{References}

Acemoglu, Daron, Asuman Ozdaglar, and Alireza Tahbaz-Salehi, "Systemic Risk and Stability in Financial Networks," American Economic Review, 2015, 105, 564-608.

_, Vasco M. Carvalho, Asuman Ozdaglar, and Alireza Tahbaz-Salehi, "The Network Origins of Aggregate Fluctuations," Econometrica, 2012, 80, 1977-2016.

Acharya, Viral V., Itamar Drechsler, and Philipp Schnabl, "A Pyrrhic Victory? Bank Bailouts and Sovereign Credit Risk," Journal of Finance, 2014, 69, 2689-2739.

Adrian, Tobias and Markus K. Brunnermeier, "CoVaR," American Economic Review, 2016, 106, 1705-1741.

_ , Richard K. Crump, and Emanuel Moench, "Pricing the Term Structure with Linear Regressions," Journal of Financial Economics, 2013, 110, 110-138.

Aldasoro, Inaki and Torsten Ehlers, "The Credit Default Swap Market: What a Difference a Decade Makes," BIS Quarterly Review, 2018, June, 1-14.

Allen, Linda, Turan G. Bali, and Yi Tang, "Does Systemic Risk in the Financial Sector Predict Future Economic Downturns?," Review of Financial Studies, 2012, 25, 3000-3036.

Alter, Adrian and Andreas Beyer, "The Dynamics of Spillover Effects during the European Sovereign Debt Turmoil," Journal of Banking and Finance, 2014, 42, 134-153.

Ando, Tomohiro and Jushan Bai, "Quantile Co-movement in Financial Markets: A Panel Quantile Model with Unobserved Heterogeneity," Journal of the American Statistical Association, 2020, 115, 266-279.

Ang, Andrew and Francis A. Longstaff, "Systemic Sovereign Credit Risk: Lessons from the U.S. and Europe," Journal of Monetary Economics, 2013, 60, 493-510.

- and Geert Bekaert, "International Asset Allocation with Regime Shifts," Review of Financial Studies, 2002, 15, 1137-1187.

Bai, Jennie and Shang-Jin Wei, "Property Rights Gaps and CDS Spreads: When is there a Strong Transfer Risk from the Sovereigns to the Corporates?, Quarterly Journal of Finance, 2017, $\%$.

Bai, Jushan and Serena Ng, "Determining the Number of Factors in Approximate Factor Models," Econometrica, 2002, 70, 191-221.

_ and _ , "Principal Components Estimation and Identification of Static Factors," Journal of Econometrics, 2013, 176, 18-29.

_ , Kunpeng Li, and Lina Lu, "Estimation and Inference of FAVAR Models," Journal of Business and Economic Statistics, 2016, 34, 620-641.

Bali, Turan G. and David Weinbaum, "A Conditional Extreme Value Volatility Estimator based on High-Frequency Returns," Journal of Economic Dynamics and Control, 2007, 31, 361-397.

Baruník, Jozef, Evžen Kočenda, and Lukáš Vácha, "Asymmetric Connectedness on the U.S. Stock Market: Bad and Good Volatility Spillovers," Journal of Financial Markets, 2016, 27, 55-78. 
Bekaert, Geert and Marie Hoerova, "The VIX, the Variance Premium and Stock Market Volatility," Journal of Econometrics, 2014, 183, 181-192.

Betz, Frank, Nikolaus Hautsch, Tuomas A. Peltonen, and Melanie Schienle, "Systemic Risk Spillovers in the European Banking and Sovereign Network," Journal of Financial Stability, 2016, 25, 206-224.

Billio, Monica, Mila Getmansky, Andrew Lo, and Loriana Pelizzon, "Econometric Measures of Connectedness and Systemic Risk in the Finance and Insurance Sectors," Journal of Financial Economics, 2012, 104, 535-559.

Blanchard, Olivier J. and Danny Quah, "The Dynamic Effects of Aggregate Demand and Supply Disturbances," American Economic Review, 1989, 79, 655-673.

- and Mark W. Watson, "Are all Business Cycles Alike?," in R.J. Gordon, ed., The American Business Cycle, Chicago: University of Chicago Press, 1986, pp. 123-160.

Blanco, Roberto, Simon Brennan, and Ian W. Marsh, "An Empirical Analysis of the Dynamic Relationship between Investment-Grade Bonds and Credit Default Swaps," Journal of Finance, 2005, 60, 2255-2281.

Bollerslev, Tim, George Tauchen, and Hao Zhou, "Expected Stock Returns and Variance Risk Premia," Review of Financial Studies, 2009, 22, 4463-4492.

Bostanci, Görkem and Kamil Yilmaz, "How Connected is the Global Sovereign Credit Risk Network?," Journal of Banking and Finance, 2020, in press.

Caporin, M., L. Pelizzon, F. Ravazzolo, and R. Rigobon, "Measuring Sovereign Contagion in Europe," Journal of Financial Stability, 2018, 34, 150-181.

Cecchetti, Stephen G. and Hong Li, "Measuring the Impact of Asset Price Booms Using Quantile Vector Autoregressions," Mimeo: Brandeis University February 2008.

Chavleishvili, Sulkhan and Simone Manganelli, "Quantile Impulse Response Functions," Mimeo: Goethe University Frankfurt January 2016.

Claeys, Peter and Bořek Vašíček, "Measuring Bilateral Spillover and Testing Contagion on Sovereign Bond Markets in Europe," Journal of Banking and Finance, 2014, 46, 151-165.

Corsi, Fulvio, "A Simple Approximate Long Memory Model of Realized Volatility," Journal of Financial Econometrics, 2009, 7, 174-196.

Demirer, Mert, Francis X. Diebold, Laura Liu, and Kamil Yilmaz, "Estimating Global Bank Network Connectedness," Journal of Applied Econometrics, 2018, 33, 1-15.

Dendramis, Yiannis, George Kapetanios, and Elias Tzavalis, "Shifts in Volatility Driven by Large Stock Market Shocks," Journal of Economic Dynamics and Control, 2015, 55, 130147.

Diebold, Francis X. and Kamil Yilmaz, "Measuring Financial Asset Return and Volatility Spillovers, with Application to Global Equity Markets," The Economic Journal, 2009, 119, 158-171.

_ and _ , "On the Network Topology of Variance Decompositions: Measuring the Connectedness of Financial Firms," Journal of Econometrics, 2014, 182, 119-134. 
Dungey, Mardi, John Harvey, and Vladimir Volkov, "The Changing International Network of Sovereign Debt and Financial Institutions," Journal of International Financial Markets, Institutions and Money, 2019, 60, 149-168.

Fama, Eugene F. and Kenneth R. French, "Common Risk Factors in the Returns on Stocks and Bonds," Journal of Financial Economics, 1993, 33, 3-56.

Farhi, Emmanuel and Jean Tirole, "Deadly Embrace: Sovereign and Financial Balance Sheets Doom Loops," Review of Economic Studies, 2018, 85, 1781-823.

Feldhütter, Peter and Mads Stenbo Nielsen, "Systematic and Idiosyncratic Default Risk in Synthetic Credit Markets," Journal of Financial Econometrics, 2012, 10, 292-324.

Fender, Ingo, Bernd Hayo, and Matthias Neuenkirch, "Daily Pricing of Emerging Market Sovereign CDS Before and During the Global Financial Crisis," Journal of Banking and Finance, 2012, 36, 2786-2794.

Fruchterman, Thomas M.J. and Edward M. Reingold, "Graph Drawing by ForceDirected Placement," Software - Practice and Experience, 1991, 21, 1129-1164.

Gabaix, Xavier, "Power Laws in Economics: An Introduction," Journal of Economic Perspectives, 2016, 30, 185-206.

Garratt, Anthony, Kevin Lee, M. Hashem Pesaran, and Yongcheol Shin, Global and National Macroeconometric Modelling: A Long-Run Structural Approach, Oxford: Oxford University Press, 2006.

Greenwood-Nimmo, Matthew J., Jingong Huang, and Viet H. Nguyen, "Financial Sector Bailouts, Sovereign Bailouts and the Transfer of Credit Risk," Journal of Financial Markets, 2019, 42, 121-142.

_, Viet H. Nguyen, and Barry Rafferty, "Risk and Return Spillovers among the G10 Currencies," Journal of Financial Markets, 2016, 31, 43-62.

Gyntelberg, Jacob, Peter Hördahl, Kristyna Ters, and Jörg Urban, "Intraday Dynamics of Euro Area Sovereign CDS and Bonds," Working Paper 423, Bank for International Settlements, Basel September 2013.

Heber, Gerd, Asger Lunde, Neil Shephard, and Kevin Sheppard, "Oxford-Man Institute's Realized Library," Dataset, Oxford-Man Institute, University of Oxford 2009.

International Monetary Fund, "Old Risks, New Challenges," Global Financial Stability Report, April 2013, IMF, Washington DC April 2013.

Jorion, Philippe and Gaiyan Zhang, "Good and Bad Credit Contagion: Evidence from Credit Default Swaps," Journal of Financial Economics, 2007, 84, 860-883.

Juodis, Arturas and Simon Reese, "The Incidental Parameters Problem in Testing for Remaining Cross-section Correlation," Working Paper 1810.03715, arXiv 2018.

Koenker, Roger and Gilbert Bassett, "Regression Quantiles," Econometrica, 1978, 46, $33-50$.

- and Kevin F. Hallock, "Quantile Regression," Journal of Economic Perspectives, 2001, $15,143-156$.

- and Zhijie Xiao, "Quantile Autoregression," Journal of the American Statistical Association, 2006, 101, 980-90. 
Linnemann, Ludger and Roland Winkler, "Estimating Nonlinear Effects of Fiscal Policy using Quantile Regression Methods," Oxford Economic Papers, 2016, 68, 1120-1145.

Londono, Juan M., "Bad Bad Contagion," Journal of Banking and Finance, 2019, in press.

Longstaff, Francis A., Jun Pan, Lasse H. Pedersen, and Kenneth J. Singleton, "How Sovereign Is Sovereign Credit Risk?," American Economic Journal: Macroeconomics, 2011, 3, 75-103.

Mastromarco, Camilla, Laura Serlenga, and Yongcheol Shin, "Modelling Technical Efficiency in Cross Sectionally Dependent Stochastic Frontier Panels," Journal of Applied Econometrics, 2016, 31, 281-297.

McNeil, Alexander J. and Rüdiger Frey, "Estimation of Tail-Related Risk Measures for Heteroskedastic Financial Time Series: An Extreme Value Approach," Journal of Empirical Finance, 2000, 7, 271-300.

Pan, Jun and Kenneth J. Singleton, "Default and Recovery Implicit in the Term Structure of Sovereign CDS Spreads," Journal of Finance, 2008, 63, 2345-2384.

Pesaran, M. Hashem, "General Diagnostic Tests for Cross Section Dependence in Panels," Working Paper 1229, CESifo 2004.

_ , "Testing Weak Cross-Sectional Dependence in Large Panels," Econometric Reviews, 2015, 34, 1089-1117.

Schüler, Yves S., "Asymmetric Effects of Uncertainty over the Business Cycle: A Quantile Structural Vector Autoregressive Approach," Working Paper 2014-02, University of Konstanz January 2014.

Sims, Christopher A., "Are Forecasting Models Usable for Policy Analysis?," Minneapolis Federal Reserve Bank Quarterly Review, 1986, Winter, 2-16.

Zellner, Arnold and Tomohiro Ando, "A Direct Monte Carlo Approach for Bayesian Analysis of the Seemingly Unrelated Regression Model," Journal of Econometrics, 2010, 159, $33-45$.

Zhu, Huiming, Xianfang Su, Yawei Guo, and Yinghua Ren, "The Asymmetric Effects of Oil Price Shocks on the Chinese Stock Market: Evidence from a Quantile Impulse Response Perspective," Sustainability, 2016, 8, 1-19. 


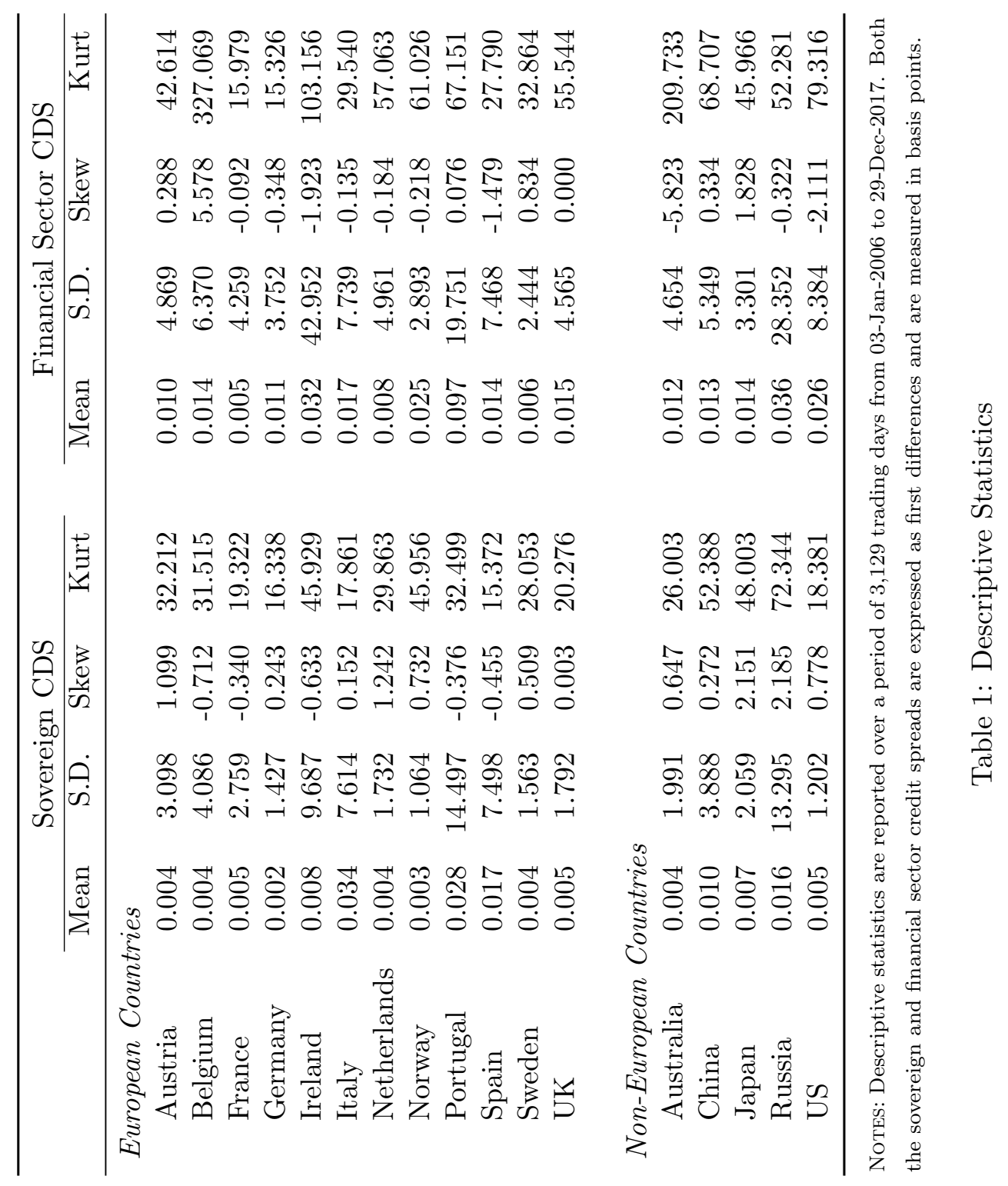




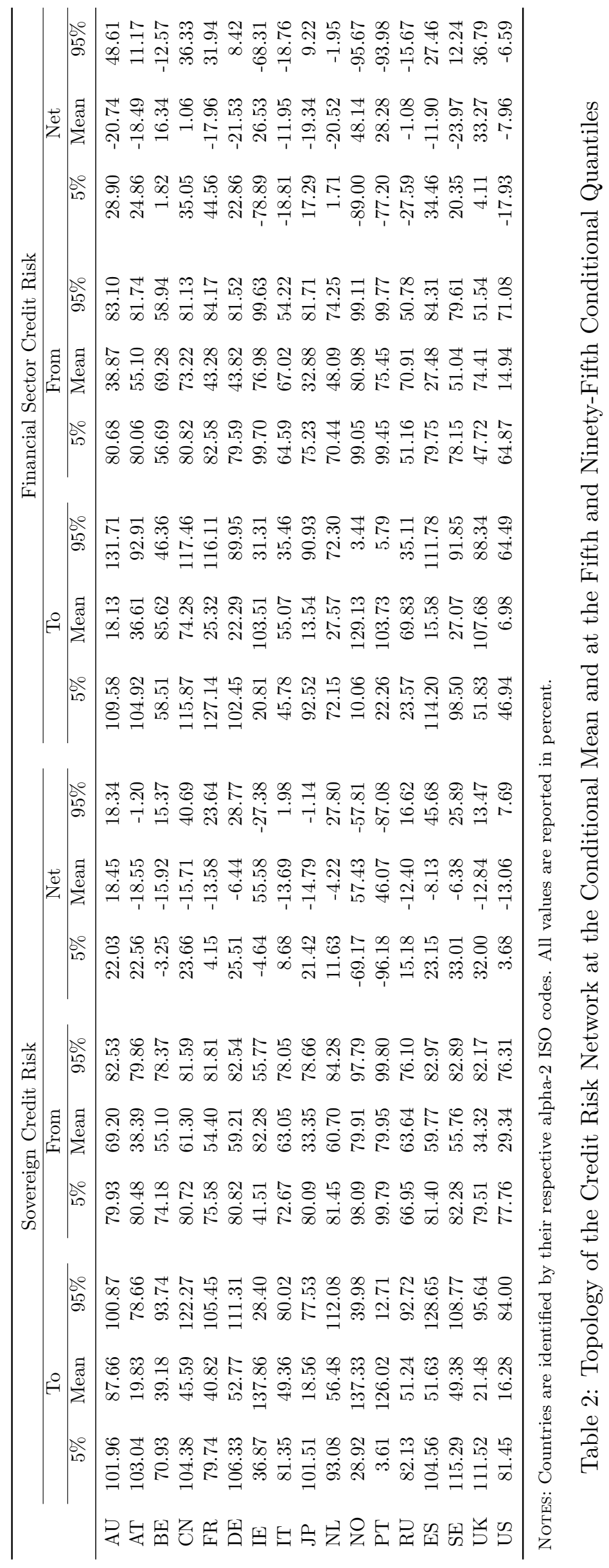




\begin{tabular}{lccccc}
\hline & Mean & $5 \%$ & Med. & $95 \%$ & RTD \\
\hline Mean & 1.000 & 0.411 & 0.719 & 0.774 & 0.438 \\
$5 \%$ & 0.411 & 1.000 & 0.329 & 0.367 & -0.417 \\
Med. & 0.719 & 0.329 & 1.000 & 0.585 & 0.317 \\
$95 \%$ & 0.774 & 0.367 & 0.585 & 1.000 & 0.693 \\
RTD & 0.438 & -0.417 & 0.317 & 0.693 & 1.000 \\
\hline
\end{tabular}

Table 3: Correlation among Spillover Indices Evaluated at Selected Quantiles

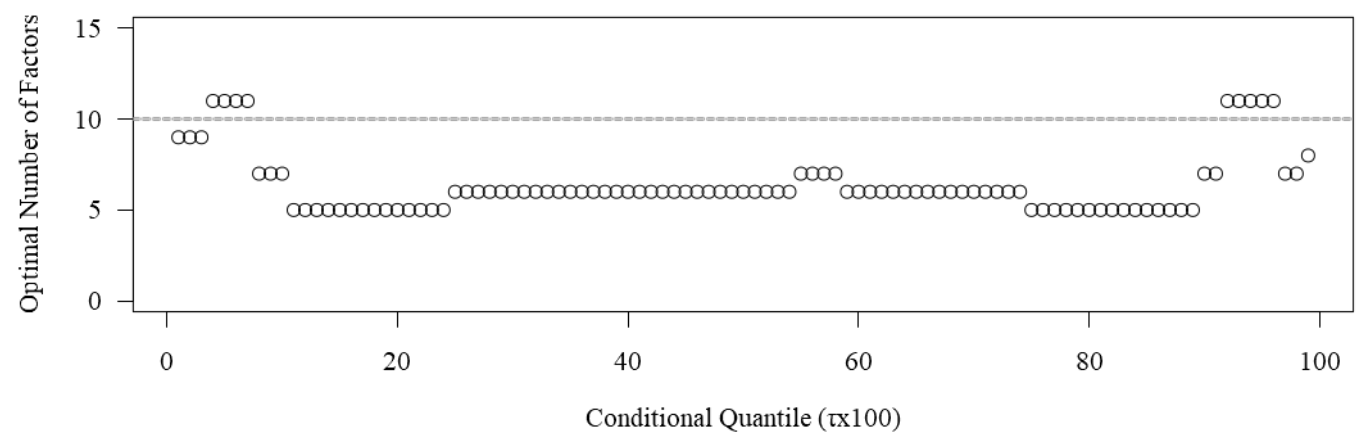

Notes: The figure reports the selected number of unobserved common factors at the $\tau$ th conditional quantile (plotted as a circle) relative to the number chosen at the conditional mean (shown by the dashed line).

Figure 1: Optimal Number of Factors at the Conditional Mean and at each Conditional Quantile

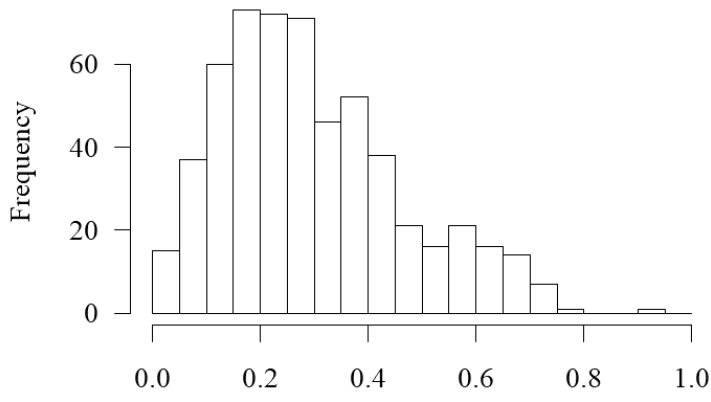

(a) Simple VAR(1) Model

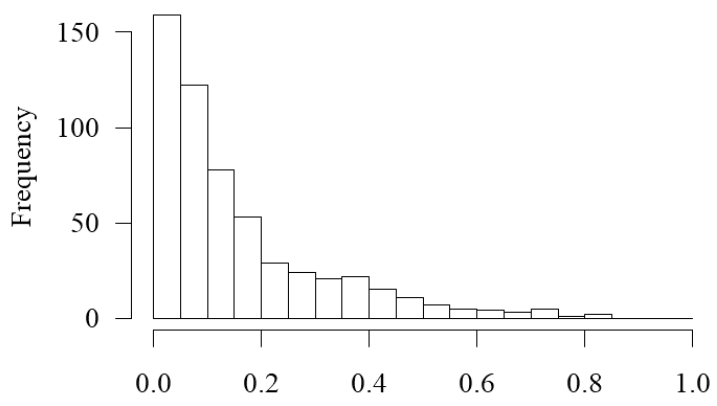

(b) Factor VAR(1) Model

Notes: The histograms show the distribution of the absolute pairwise correlations between the residuals of the simple VAR(1) model with no factor structure and our factor VAR(1) model evaluated at the conditional mean.

Figure 2: Comparison of Absolute Residual Correlations, with and without Factors 


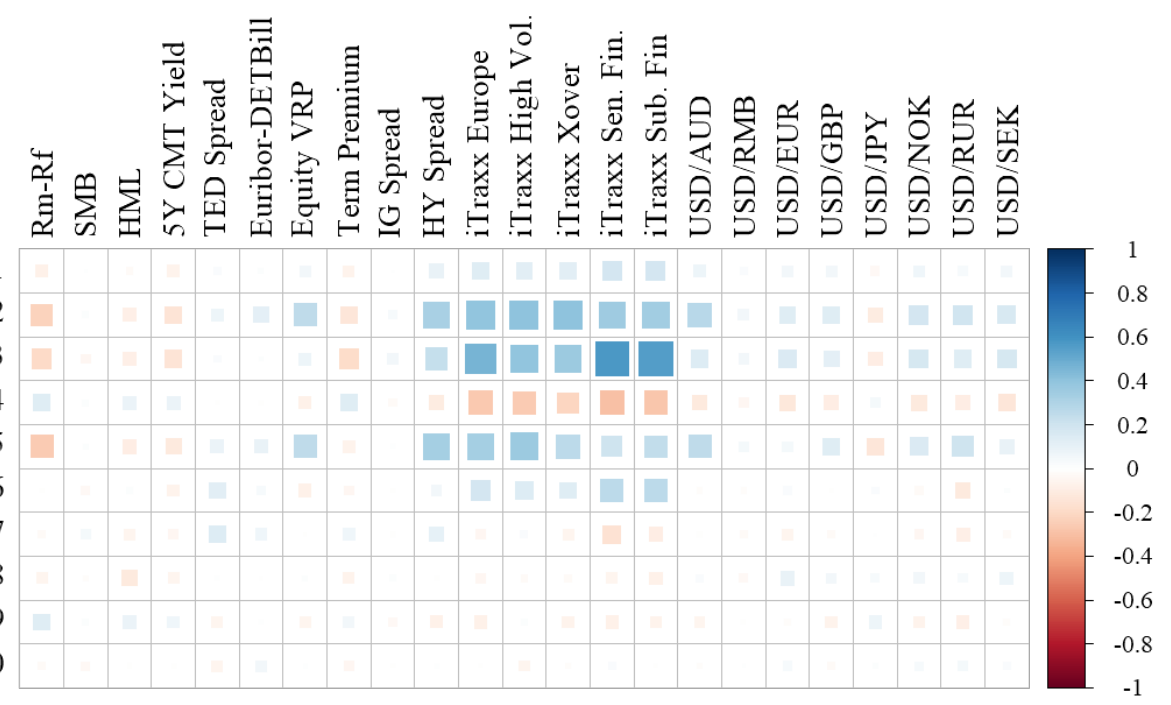

(a) Conditional Mean

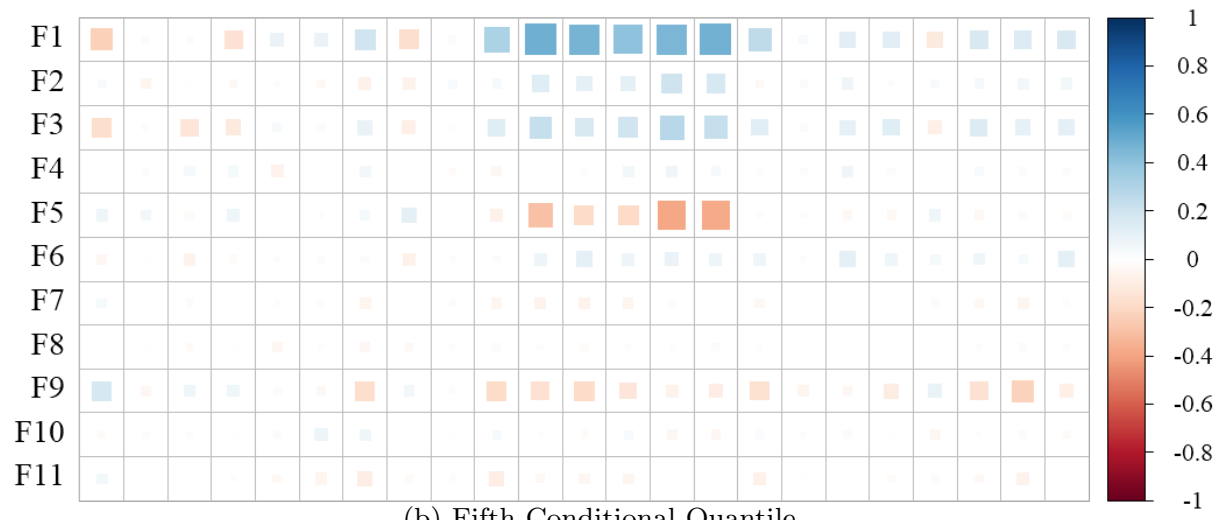

(b) Fifth Conditional Quantile

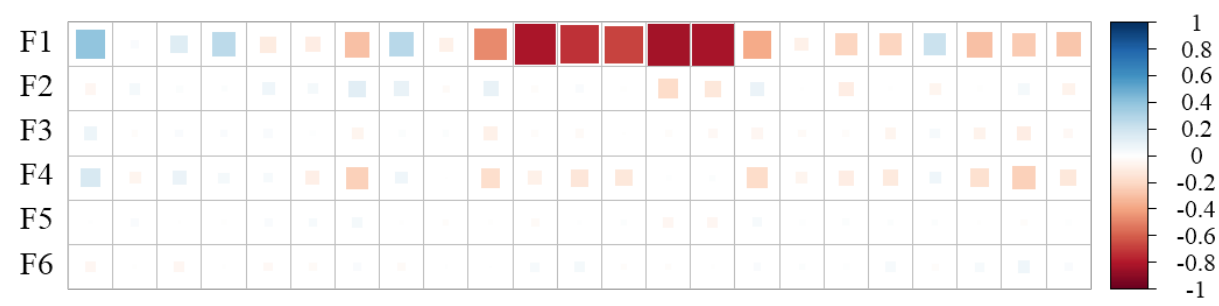

(c) Conditional Median

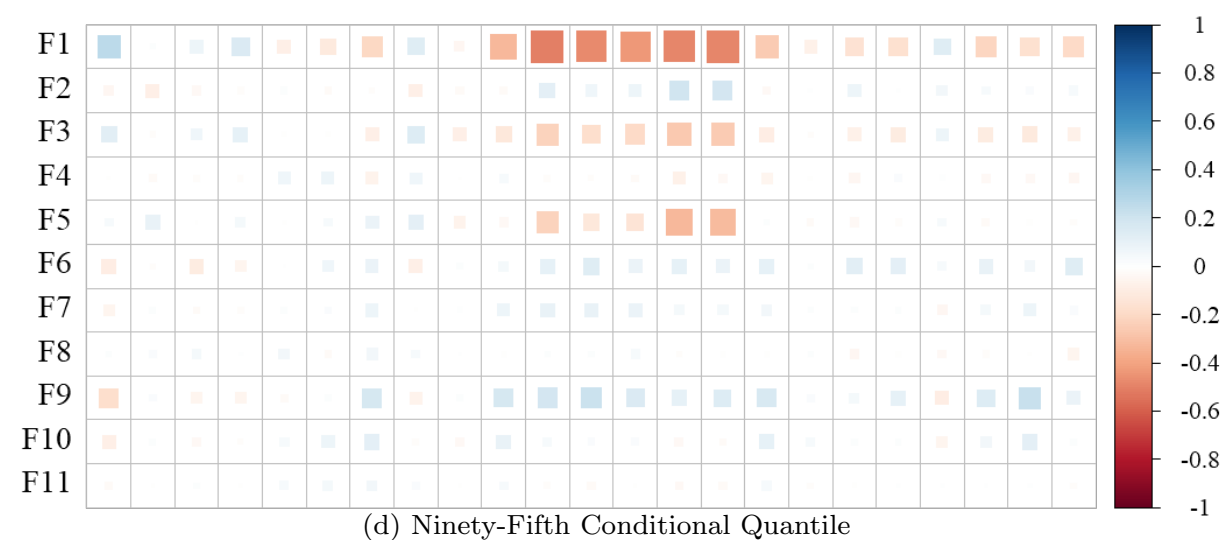

Notes: The figure reports the correlation between each of the unobserved factors selected at the conditional mean and the 5 th, 50 th and 95 th conditional quantiles (labeled ' $\mathrm{F} 1$ ', ' $\mathrm{F} 2$ ',,. ) and the set of 23 observed global variables described in Section 4.1.

Figure 3: Relation of Unobserved Factors to Observed Global Variables 


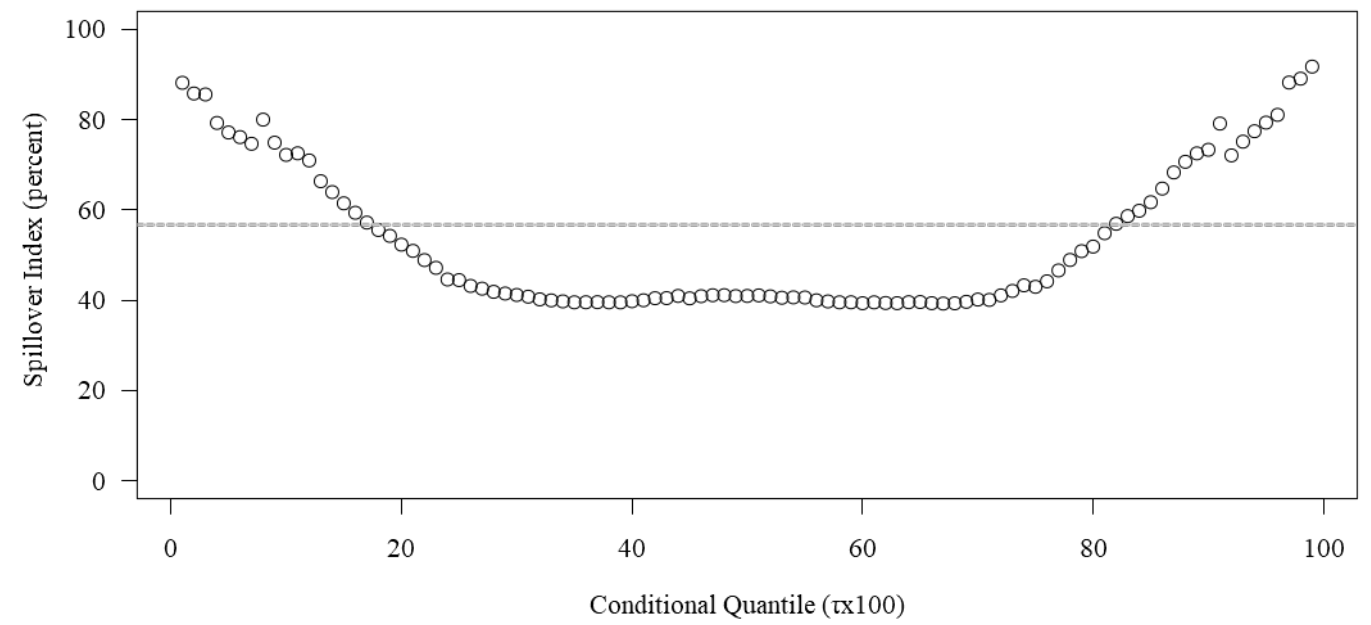

Notes: The figure reports the value of the spillover index defined in (20) evaluated at the $\tau$ th conditional quantile (plotted as a circle) relative to the value at the conditional mean (shown by the dashed line).

Figure 4: Variation in the DY Spillover Index over the Conditional Distribution 

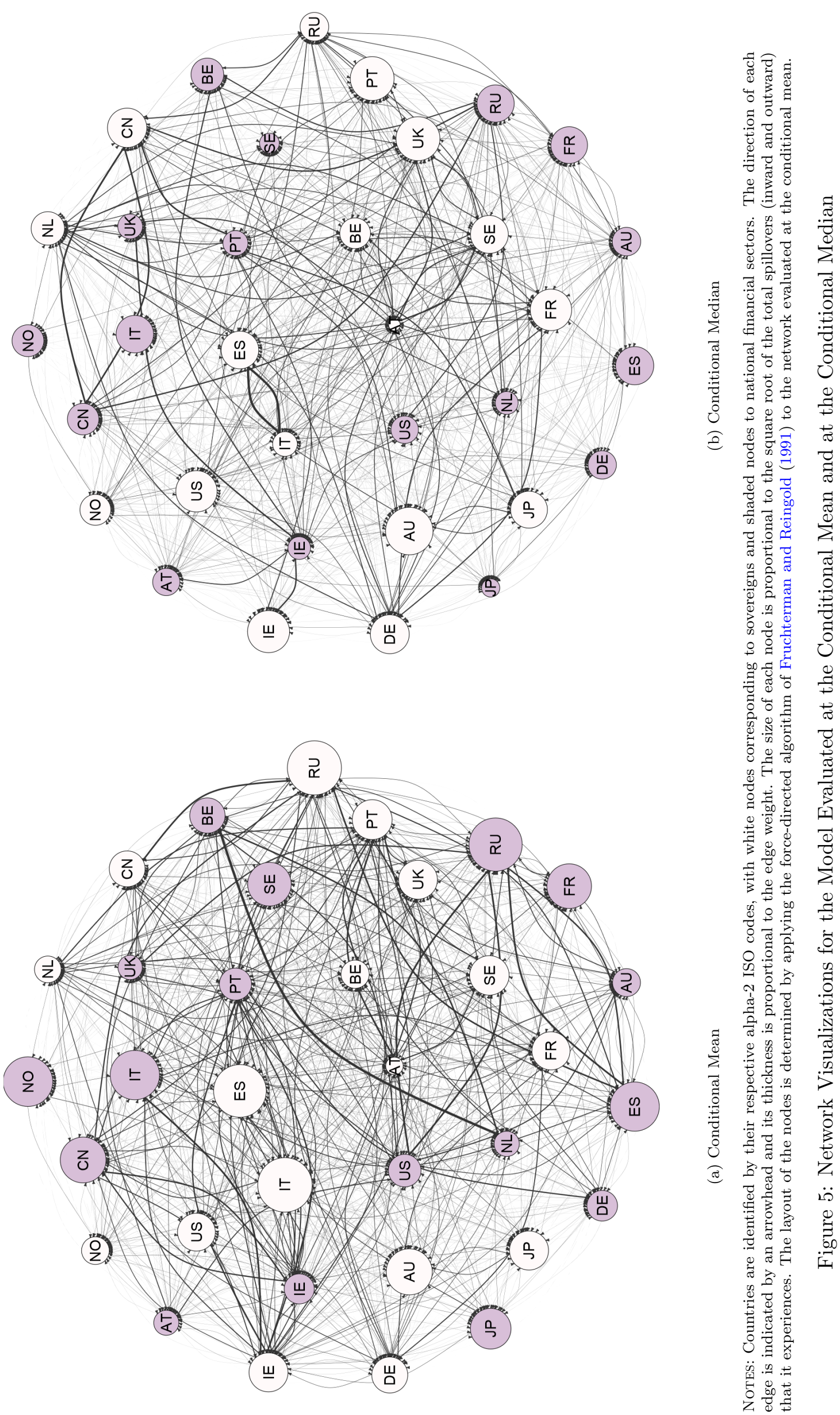

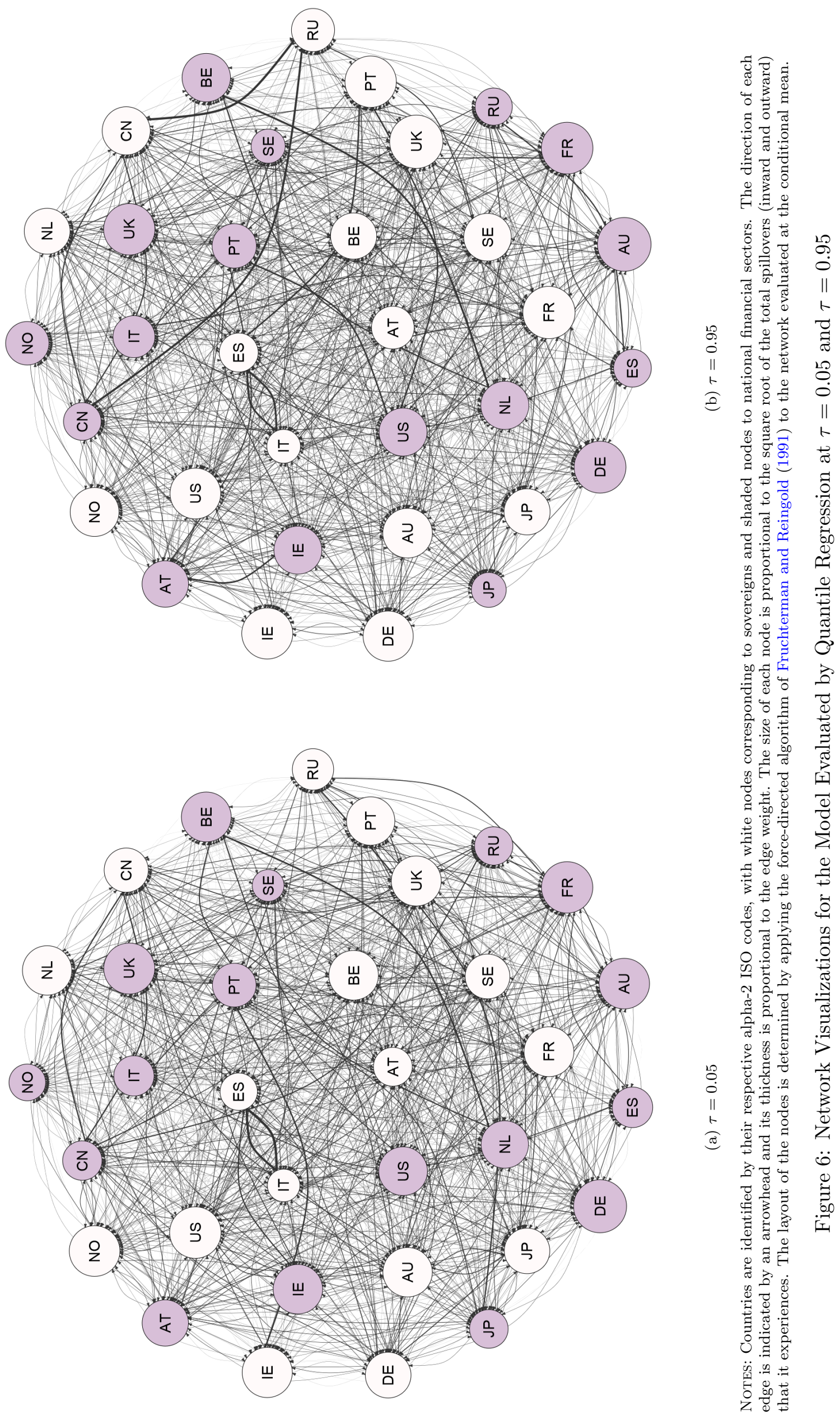


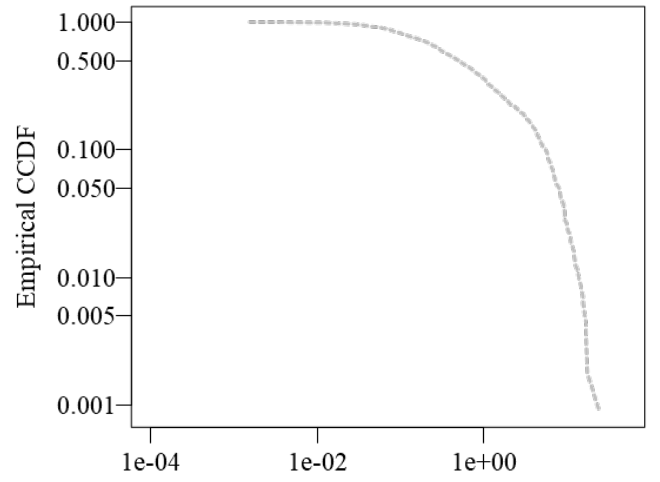

(a) Conditional Mean

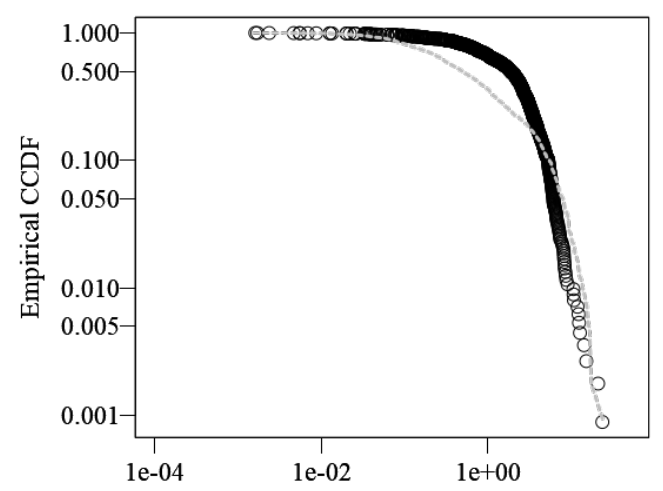

(c) $\tau=0.05$

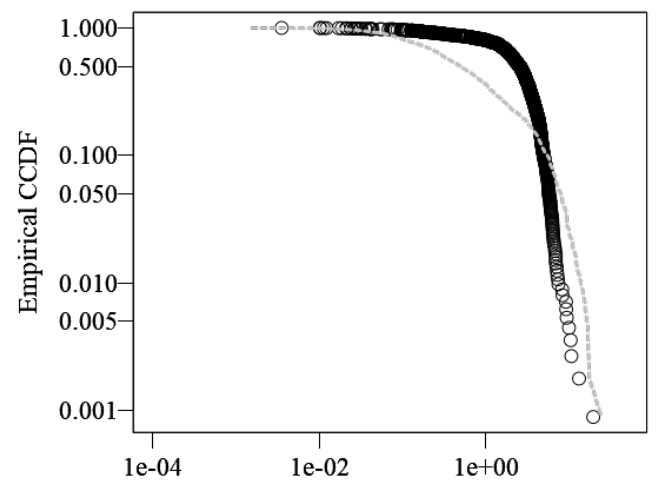

(e) $\tau=0.01$

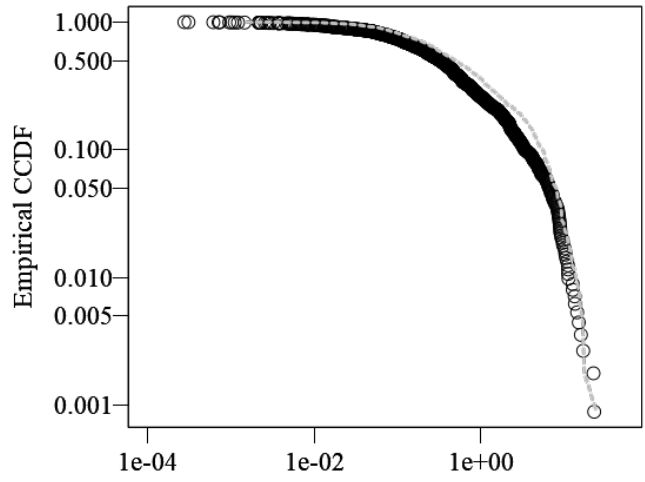

(b) Conditional Median

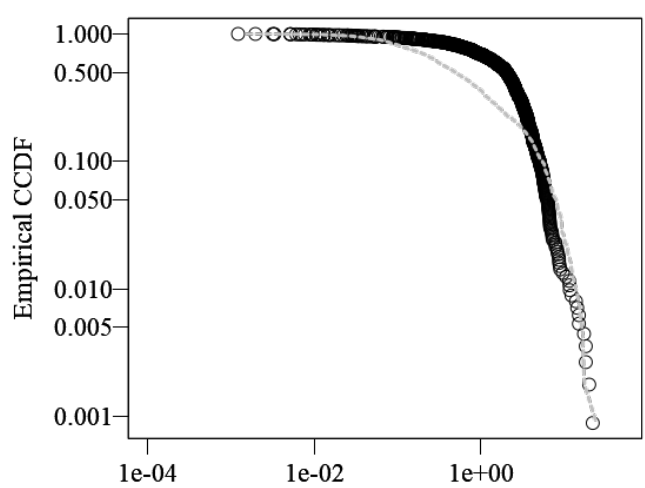

(d) $\tau=0.95$

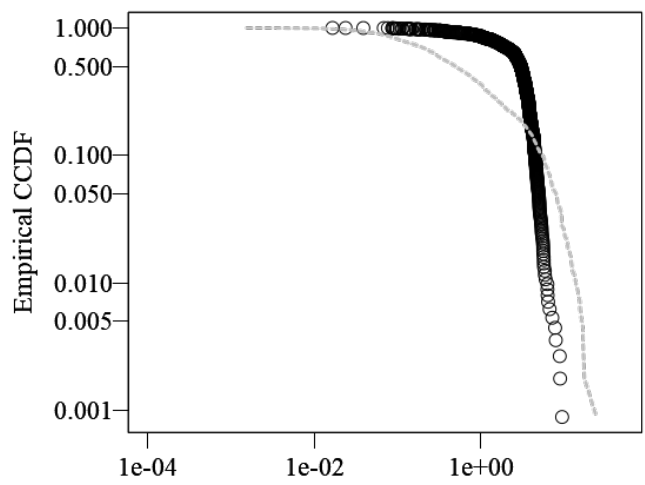

(f) $\tau=0.99$

Notes: The figure reports the empirical counter cumulative distribution function $(\mathrm{CCDF})$ of the $m(m-1)$ offdiagonal elements of the adjacency matrix on a logarithmic scale. The CCDF for the model evaluated at the conditional mean is shown as a dashed line in every panel for comparison.

Figure 7: Quantile Variation in the Shape of the Spillover Density 

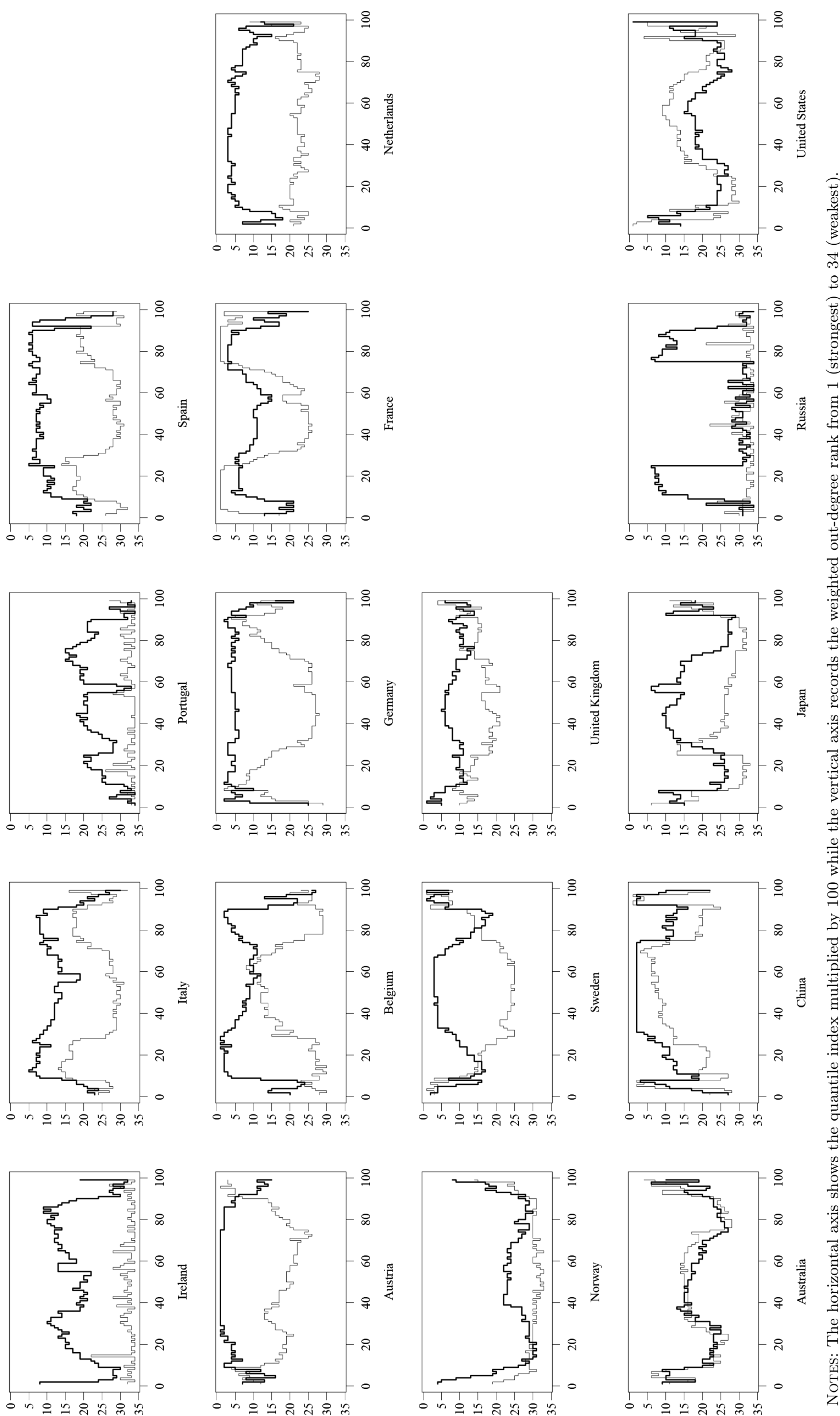

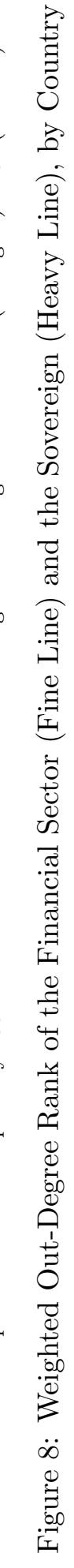



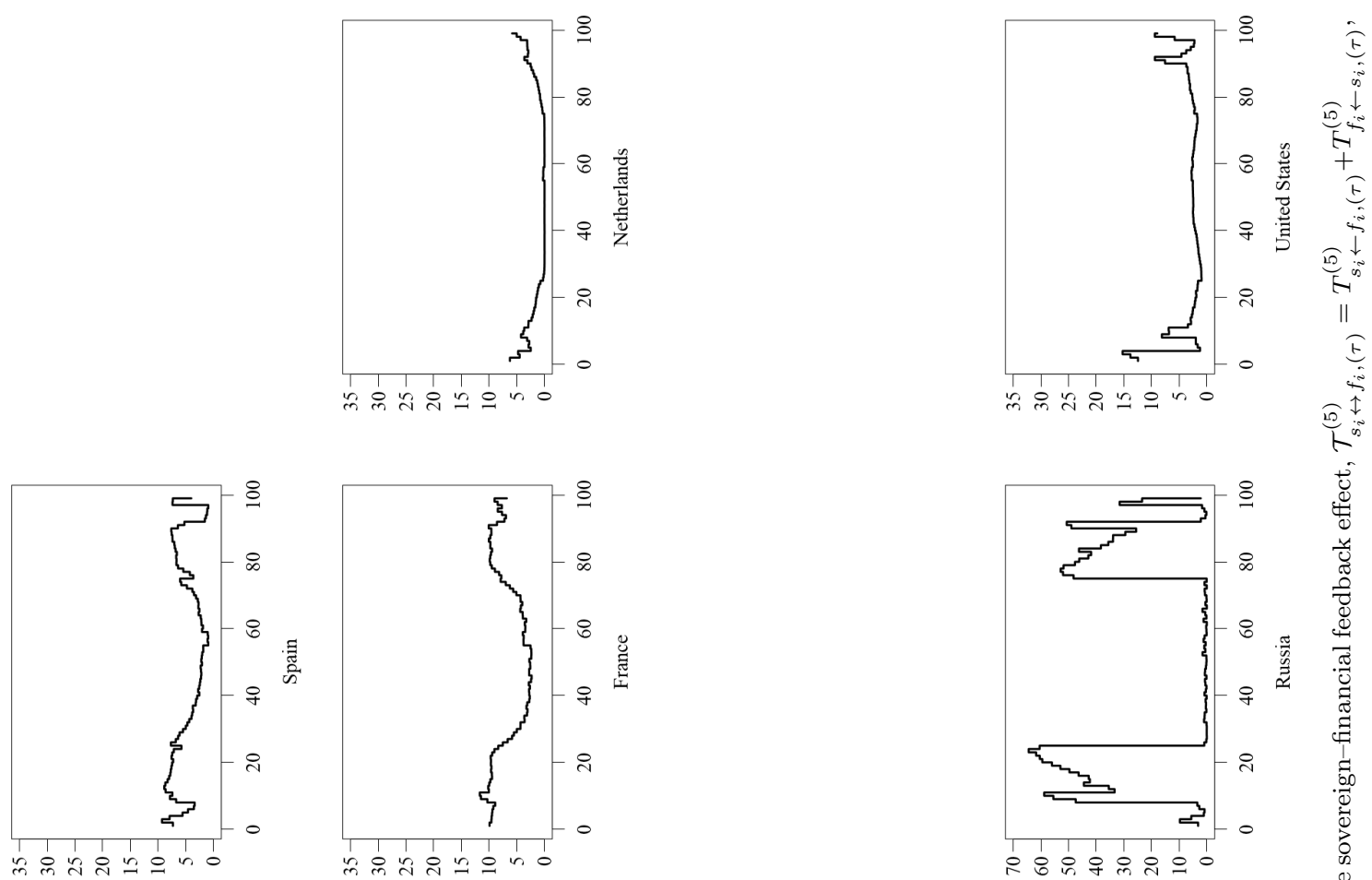

D
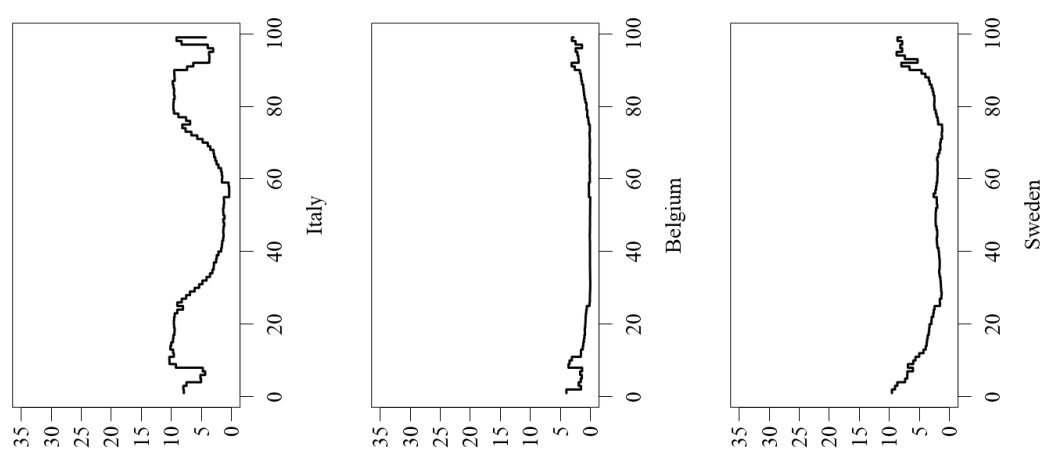

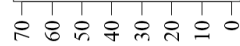
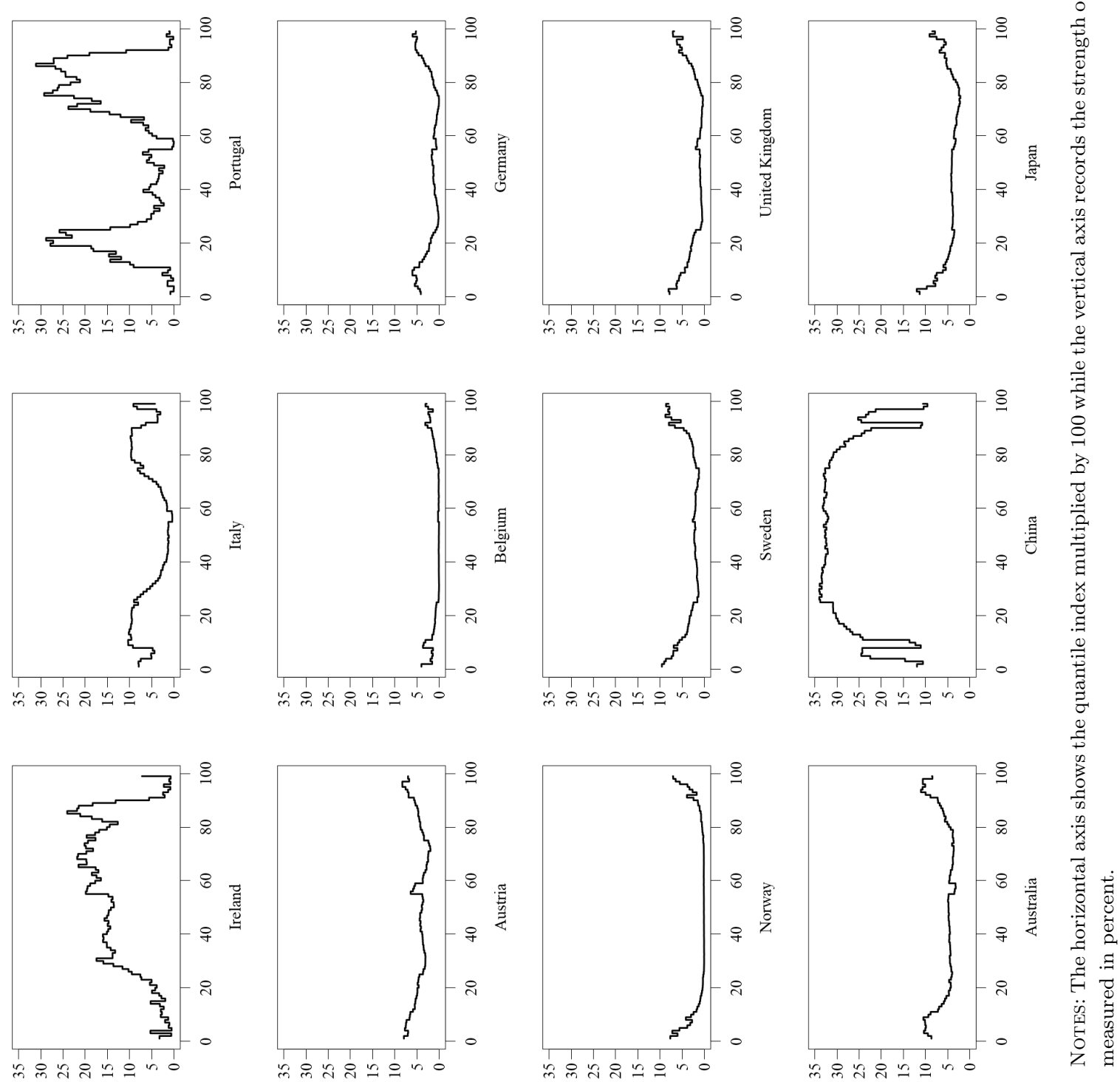

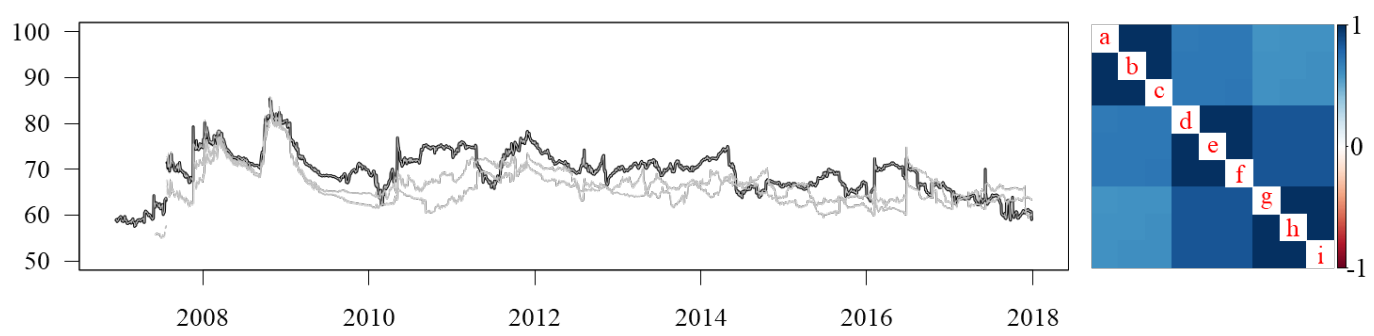

(a) Conditional Mean
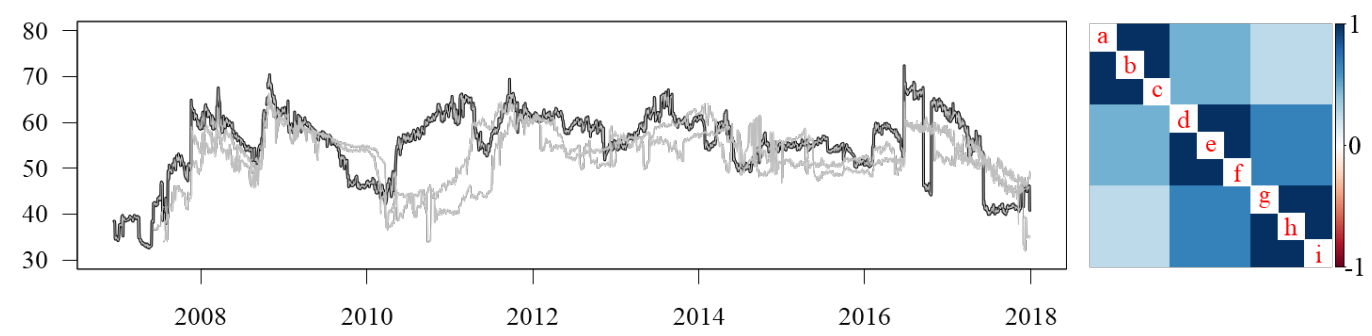

(b) Conditional Median
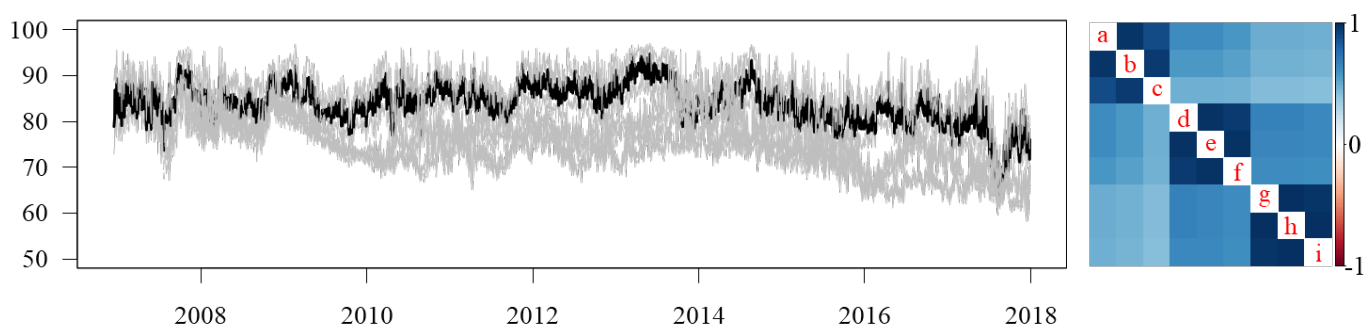

(c) Fifth Conditional Quantile
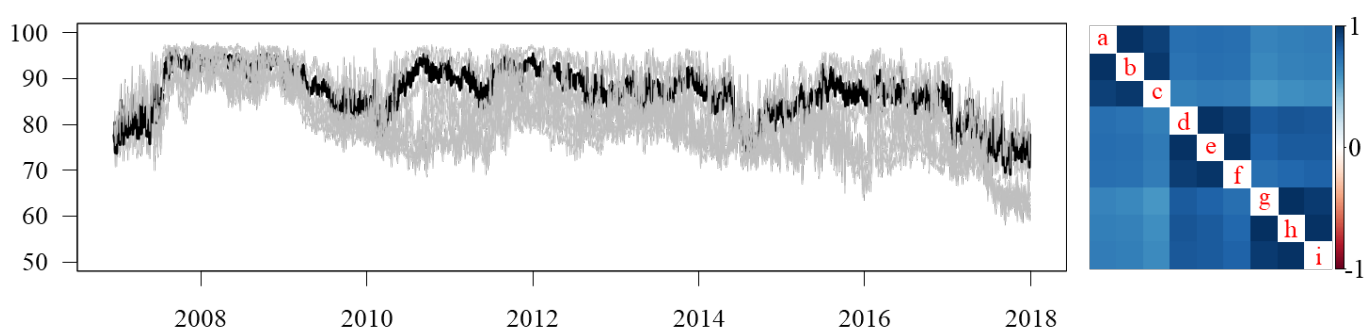

(d) Ninety-Fifth Conditional Quantile

NotEs: In each panel, results for our baseline setting with $w=250$ and $h=5$ trading days are shown as a heavy black line. Results for each other combination of $w \in\{250,375,500\}$ and $h \in\{3,5,10\}$ trading days are shown as fine gray lines. Letters $\mathrm{a}-\mathrm{i}$ in the common-sample correlation heatmaps refer to the following combinations: (a) $w=250, h=3$; (b) $w=250, h=5$; (c) $w=250, h=10$; (d) $w=375, h=3$; (e) $w=375$, $h=5$; (f) $w=375, h=10$; (g) $w=500, h=3$; (h) $w=500, h=5$; and (i) $w=500, h=10$.

Figure 10: Robustness to the Choice of Rolling Window and Forecast Horizon 


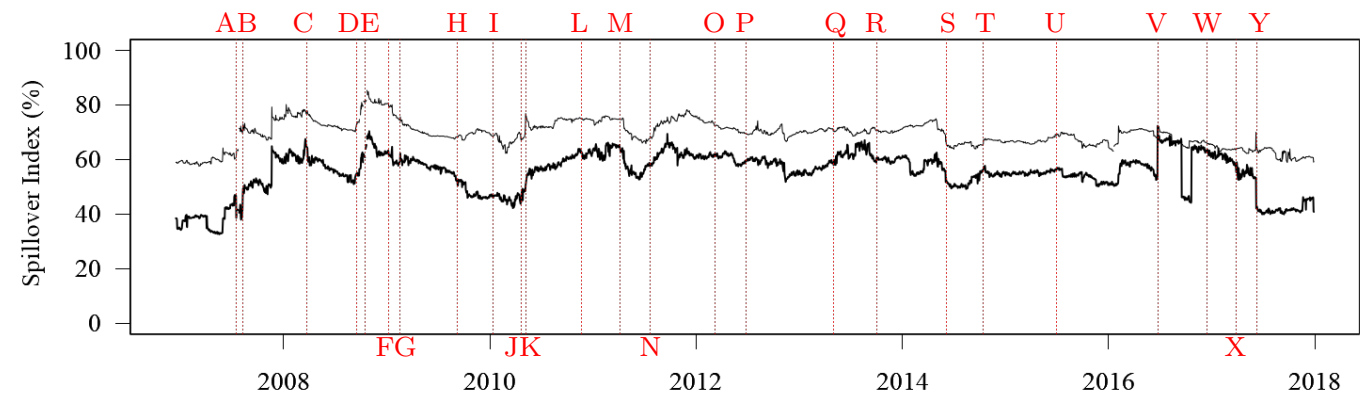

(a) Conditional Mean and Median

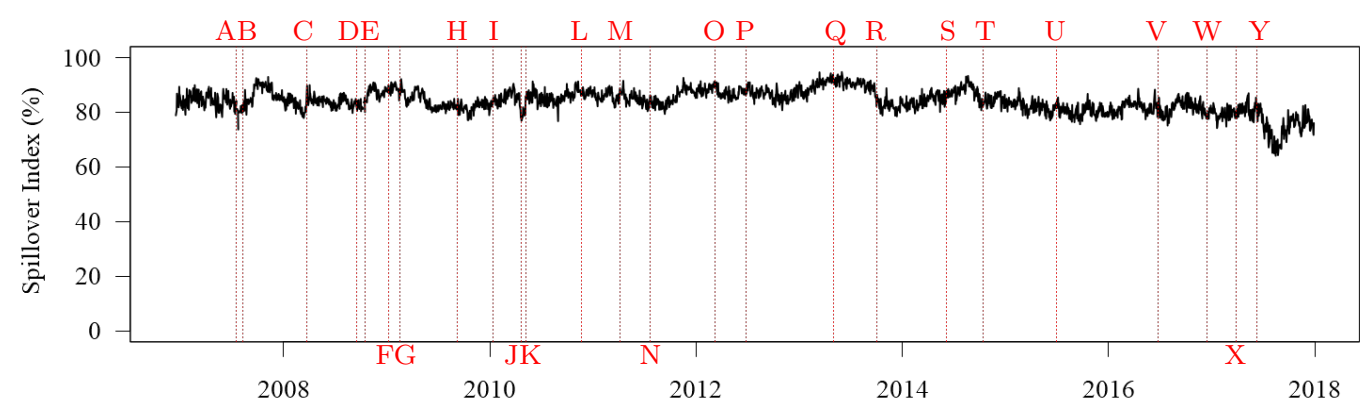

(b) Fifth Conditional Quantile

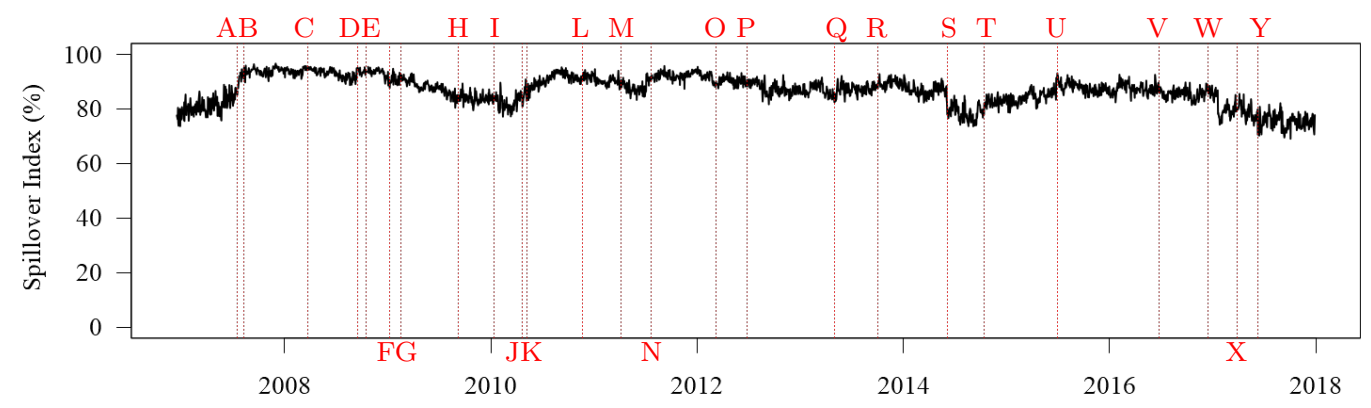

(c) Ninety-Fifth Conditional Quantile

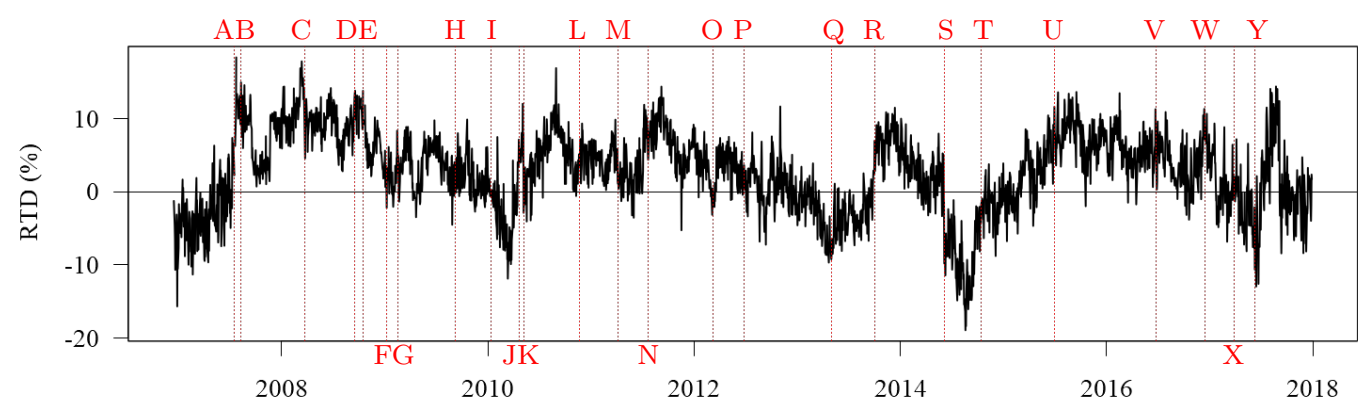

(d) Relative Tail-Dependence

NOTES: The figure reports the value of the spillover index defined in (20) evaluated at the mean (shown as a fine line in panel (a)) and at the 5th, 50th and 95th conditional quantiles (shown as heavy lines in panels (a)-(c)) as well as the difference between the spillover index at the 95th and 5th conditional quantiles (shown as a heavy line in panel (d)). The results are obtained from rolling regressions with a window length of 250 trading days. The date shown corresponds to the last day of each rolling window. We suppress eight rolling samples where the system exhibits instability at the conditional mean. The following events are marked: A: Bear Stearns announces hedge fund losses (17-Jul-07); B: BNP Paribas halts redemptions on three investment funds (09-Aug-07); C: Bear Stearns is acquired by JP Morgan (24-Mar-08); D: Lehman Brothers files for bankruptcy (15-Sep-08); E: the Troubled Asset Relief Program is announced (14-Oct-08); F: the Fed begins purchasing mortgage-backed securities issued by Fannie Mae and Freddie Mac (05-Jan-09); G: signing of the American Recovery and Reinvestment Act (17-Feb-09); H: Greek parliament is dissolved (08-Sep-09); I: report on the falsification of Greek data released (12-Jan-10); J: Greece requests aid (23-Apr-10); K: the European Financial Stability Facility is announced (09May-10); L: Ireland requests aid (22-Nov-10); M: Portugal requests aid (06-Apr-11); N: second Greek bailout (22-Jul-11); O: Greek debt swap arrangement agreed (07-Mar-2012); P: Spain requests aid (25-Jun-2012); Q: ECB cuts interest rates to a record low of 0.5\% (01-May-2013); R: US government shutdown (01-Oct-2013); S: ECB announces negative interest rate policy (05-Jun-2014); T: October 2014 flash crash (15-Oct-2014); U: Greece fails to meet its IMF payment schedule (30-Jun-2015); V: Brexit referendum (23-Jun-2016); W: Federal Reserve raises interest rates (14-Dec-2016); X: UK invokes article 50 of the Lisbon Treaty (29-Mar-2017); and Y: snap election held in the UK (08-Jun-2017).

Figure 11: Time-Varying Dependence at Selected Quantiles 


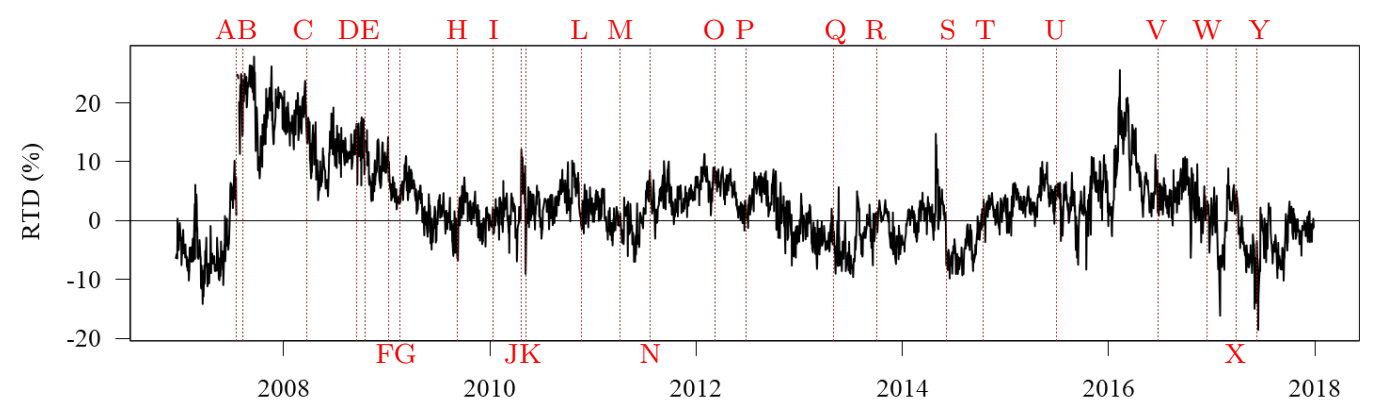

(a) $10 \%$ Relative Tail-Dependence, $S_{0.90}^{(5)}-S_{0.10}^{(5)}$

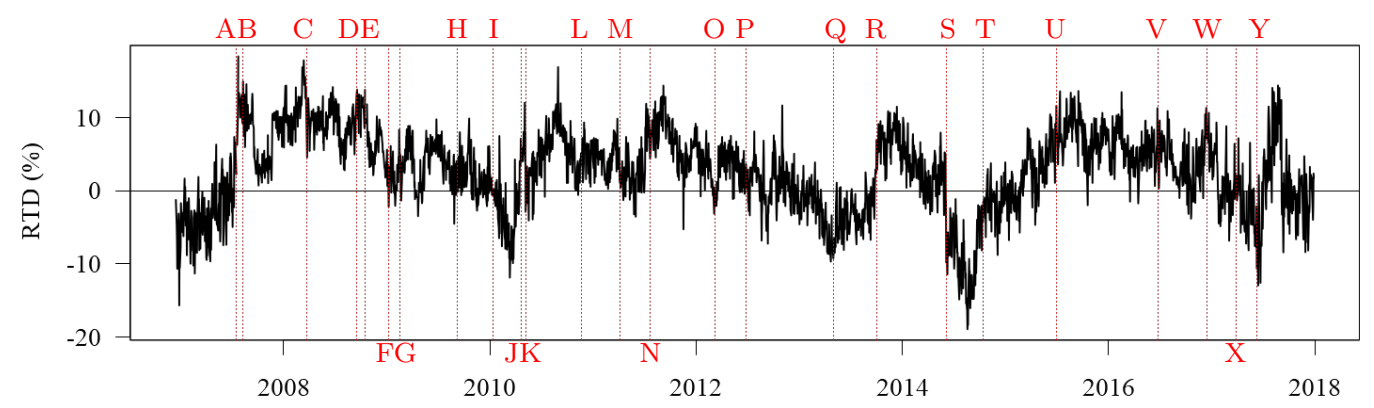

(b) $5 \%$ Relative Tail-Dependence, $S_{0.95}^{(5)}-S_{0.05}^{(5)}$

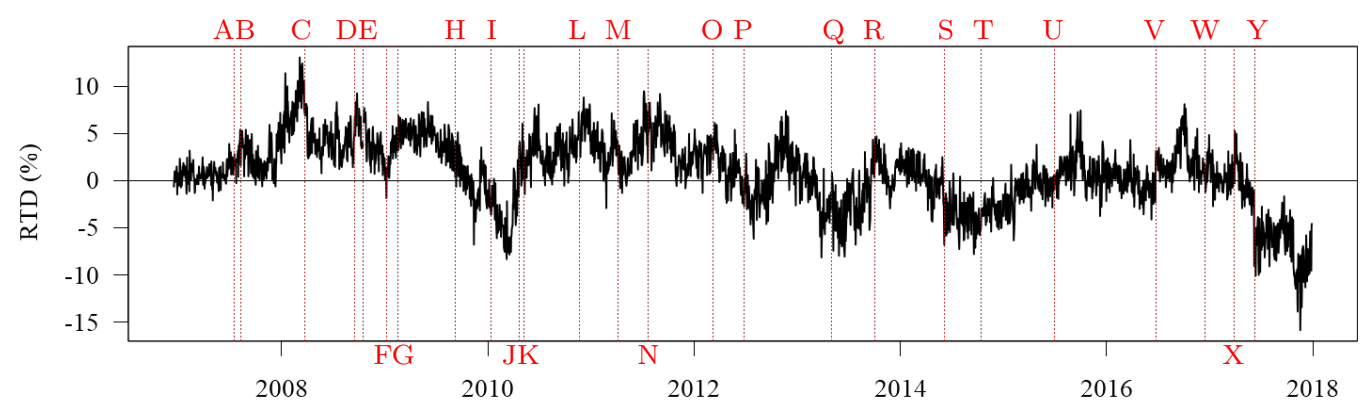

(c) $1 \%$ Relative Tail-Dependence, $S_{0.99}^{(5)}-S_{0.01}^{(5)}$

Notes: The results are obtained from rolling regressions with a window length of 250 trading days. The date shown corresponds to the last day of each rolling window. We suppress eight rolling samples where the system exhibits instability at the conditional mean. The following events are marked: A: Bear Stearns announces hedge fund losses (17-Jul-07); B: BNP Paribas halts redemptions on three investment funds (09-Aug-07); C: Bear Stearns is acquired by JP Morgan (24-Mar-08); D: Lehman Brothers files for bankruptcy (15-Sep-08); E: the Troubled Asset Relief Program is announced (14-Oct-08); F: the Fed begins purchasing mortgage-backed securities issued by Fannie Mae and Freddie Mac (05-Jan-09); G: signing of the American Recovery and Reinvestment Act (17-Feb-09); H: Greek parliament is dissolved (08-Sep-09); I: report on the falsification of Greek data released (12-Jan-10); J: Greece requests aid (23-Apr-10); K: the European Financial Stability Facility is announced (09-May-10); L: Ireland requests aid (22-Nov-10); M: Portugal requests aid (06-Apr-11); N: second Greek bailout (22-Jul-11); O: Greek debt swap arrangement agreed (07-Mar-2012); P: Spain requests aid (25-Jun2012); Q: ECB cuts interest rates to a record low of 0.5\% (01-May-2013); R: US government shutdown (01-Oct-2013); S: ECB announces negative interest rate policy (05-Jun-2014); T: October 2014 flash crash (15-Oct-2014); U: Greece fails to meet its IMF payment schedule (30-Jun-2015); V: Brexit referendum (23-Jun-2016); W: Federal Reserve raises interest rates (14-Dec-2016); X: UK invokes article 50 of the Lisbon Treaty (29-Mar-2017); and Y: snap election held in the UK (08-Jun-2017).

Figure 12: Alternative Measures of Relative Tail-Dependence 


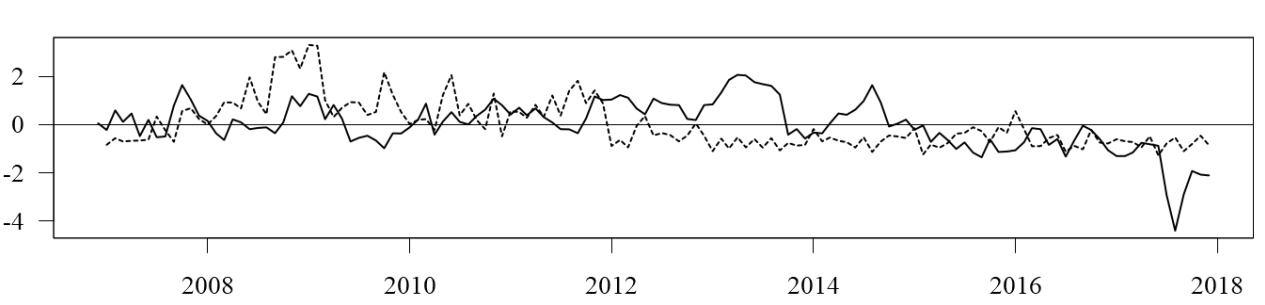

(a) Standardized CATFIN vs. Standardized Spillover Index at the 5th Conditional Quantile
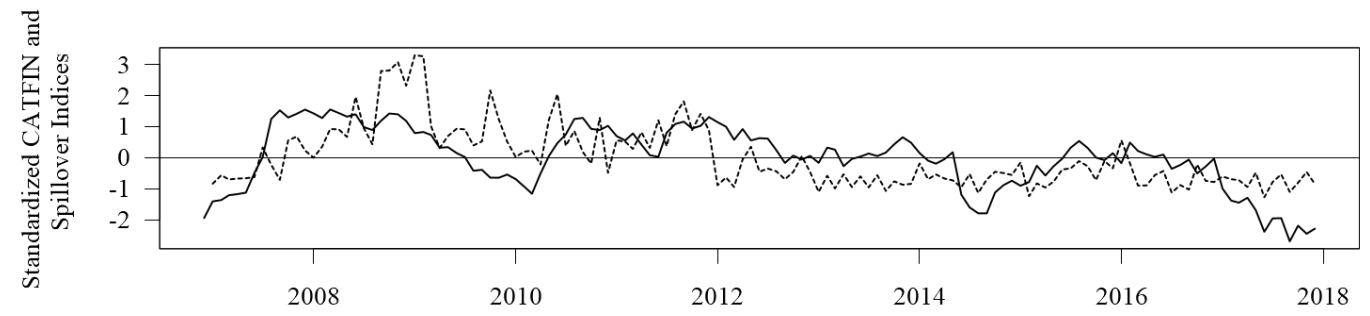

(b) Standardized CATFIN vs. Standardized Spillover Index at the 95th Conditional Quantile
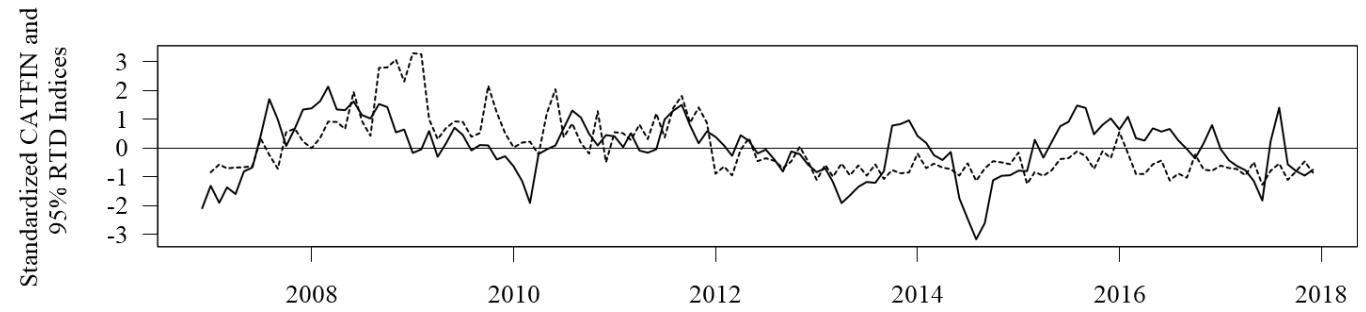

(c) Standardized CATFIN vs. Standardized 5\% RTD

NotES: CATFIN is only available at monthly frequency, so we convert our indices to monthly frequency by taking the period-average. Furthermore, to ensure that all variables are measured on a comparable scale, we standardize by subtracting the mean and dividing by the standard deviation in each case. The standardized CATFIN is shown as a dashed line and the spillover index/RTD as a solid line in each panel.

Figure 13: Comovement with the CATFIN Index 


\section{Appendix A: Robustness to the Inclusion of Greece}

To investigate the extent to which our estimation results are robust to the inclusion of Greece, we re-estimate the model including Greek data over the available sample period (03-Jan-2006 to 14-Feb-2012, shortly after which Greek sovereign CDS ceased trading on a running spread due to the expectation of an imminent credit event).

Figures A1 and A2 offer a concise summary of the key findings:

(i) In Figure A1, we replicate Figure 4 for the model including Greece. The results are remarkably similar in both cases.

(ii) In Figure A2, we replicate the rolling sample analysis in Figure 11 over the shorter sample period. For this exercise, we set the window length to 250 trading days and the forecast horizon to 5 trading days. Once again, this change in specification does not cause any substantial change in our results. The dynamic behavior of the Diebold-Yilmaz spillover index evaluated at different quantiles is comparable to our baseline specification with 17 countries. Likewise, the dynamic evolution of the 5\% RTD does not change substantially relative to the baseline model - it exhibits the same principal turnings points and shows a comparable pattern of positive and negative values.

The results of additional robustness exercises are available from the authors on request.

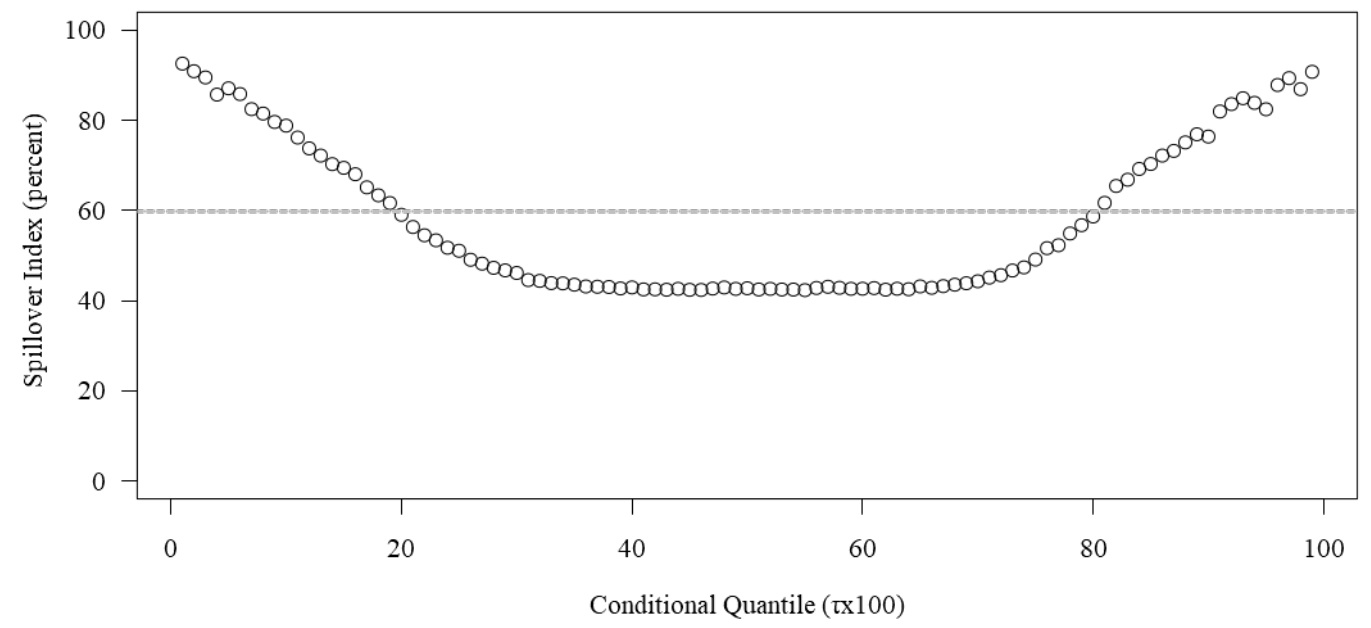

Notes: The figure reports the value of the spillover index defined in (20) evaluated at the $\tau$ th conditional quantile (plotted as a circle) relative to the value at the conditional mean (shown by the dashed line) for the model including Greece estimated using a sample ending on February 14, 2012.

Figure A1: Quantile Variation in the DY Spillover Index for the Model Including Greece 


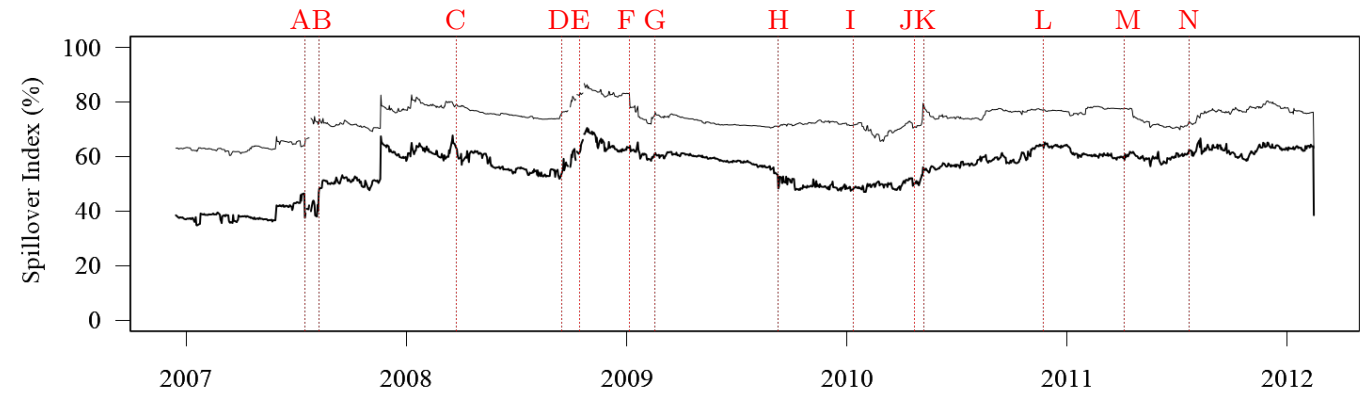

(a) Conditional Mean and Median

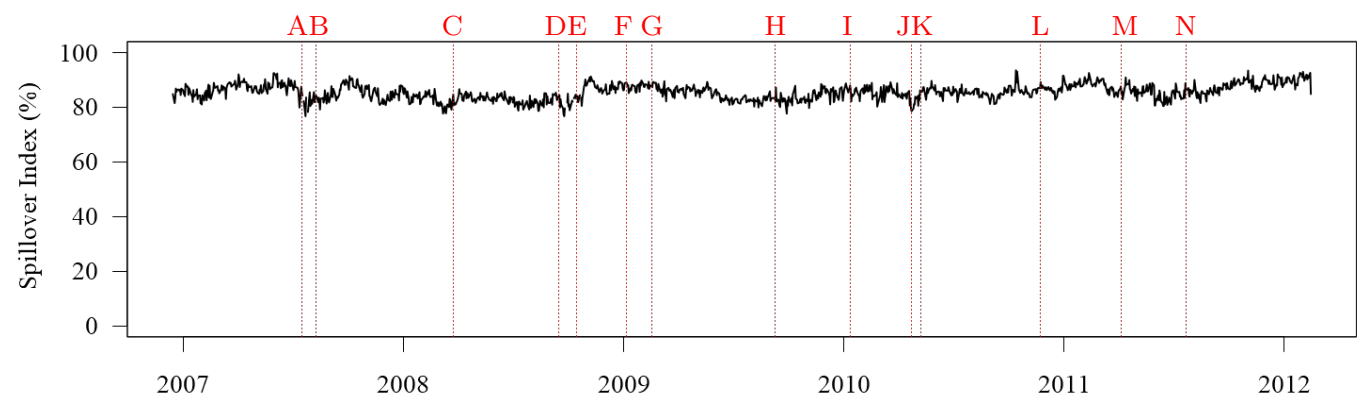

(b) Fifth Conditional Quantile

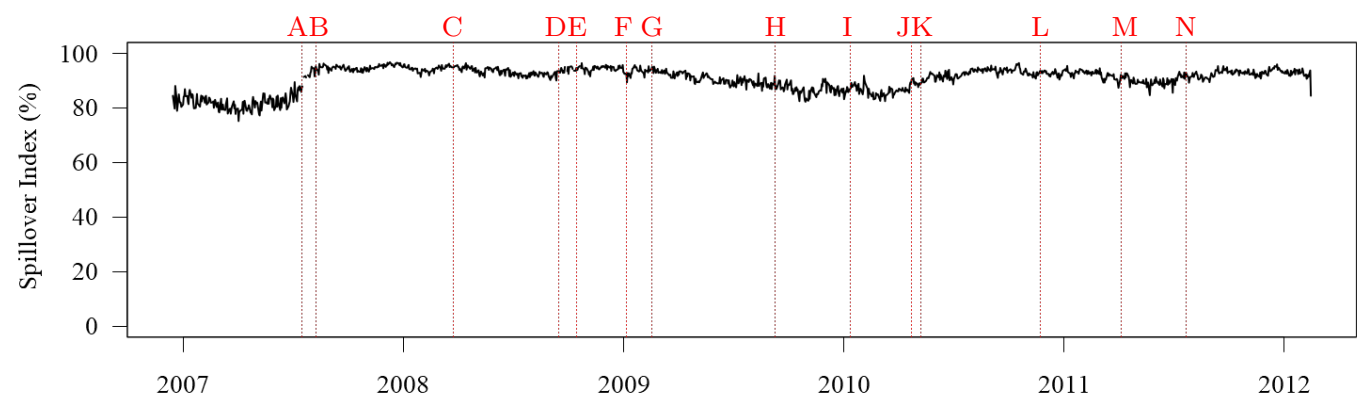

(c) Ninety-Fifth Conditional Quantile

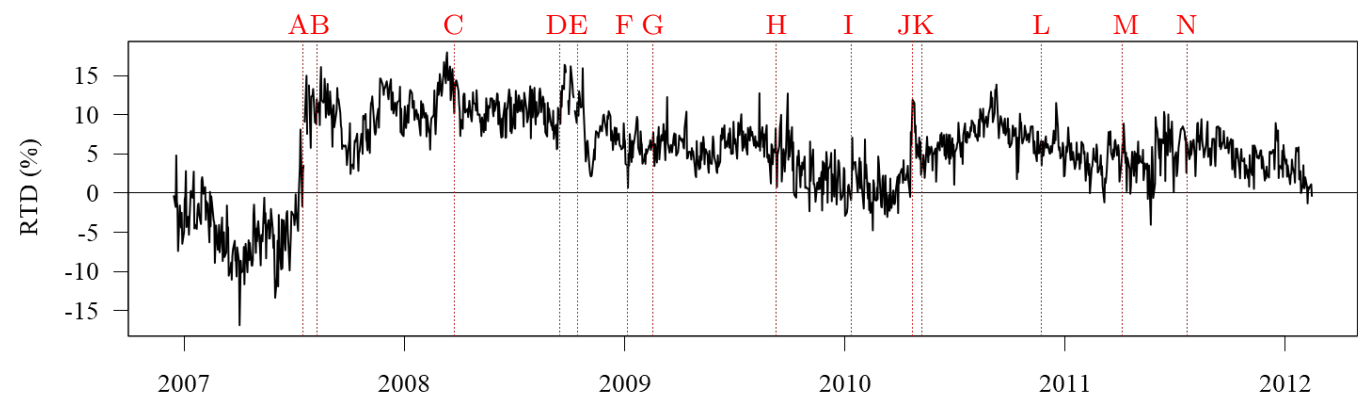

(d) Relative Tail-Dependence

NOTES: The figure reports the value of the spillover index defined in (20) evaluated at the mean (shown as a fine line in panel (a)) and at the 5th, 50th and 95th conditional quantiles (shown as heavy lines in panels (a)-(c)) as well as the difference between the spillover index at the 95th and 5th conditional quantiles (shown as a heavy line in panel (d)). The results are obtained from rolling regressions with a window length of 250 trading days. The date shown corresponds to the last day of each rolling window. We suppress four rolling samples where the system exhibits instability at the conditional mean. The following events are marked: A: Bear Stearns announces hedge fund losses (17-Jul-07); B: BNP Paribas halts redemptions on three investment funds (09-Aug-07); C: Bear Stearns is acquired by JP Morgan (24-Mar-08); D: Lehman Brothers files for bankruptcy (15-Sep-08); E: the Troubled Asset Relief Program is announced (14-Oct-08); F: the Fed begins purchasing mortgage-backed securities issued by Fannie Mae and Freddie Mac (05-Jan-09); G: signing of the American Recovery and Reinvestment Act (17-Feb-09); H: Greek parliament is dissolved (08-Sep-09); I: report on the falsification of Greek data released (12-Jan-10); J: Greece requests aid (23-Apr-10); K: the European Financial Stability Facility is announced (09-May-10); L: Ireland requests aid (22-Nov-10); M: Portugal requests aid (06-Apr-11); and N: second Greek bailout (22-Jul-11).

Figure A2: Time-Varying Dependence at Selected Quantiles for the Model Including Greece 


\title{
Appendix B: Robustness to Allowing the Number of Common Factors to Change over Rolling Samples
}

Recall that our baseline estimation results are obtained by selecting the optimal number of common factors at the conditional mean and at each conditional quantile over the full sample period and then keeping the number of factors fixed at this value throughout our rolling regressions. To test whether the assumption that the number of common factors does not change between rolling samples affects our estimation results, we re-estimate the model allowing the number of common factors at the conditional mean and at each conditional quantile to be selected optimally in each rolling sample.

In Figures B1 and B2, we compare the baseline values of the 5\% RTD and the DY spillover index at the conditional mean, the conditional median, the fifth and the ninety-fifth conditional quantiles obtained assuming a fixed number of common factors (our baseline case) against their counterparts computed allowing for a time-varying number of common factors. In each case and across the large majority of rolling samples, the results are very similar, with only minor deviations between the two lines shown in each panel of the figures. The most notable deviation between the two sets of results arises in mid-2017, where the decline in left tail connectedness observed in our baseline setting does not occur in the model in which the number of common factors is time-varying. This occurs late in our sample and does not affect our narrative. No other significant deviations occur over our sample period. This indicates that our use of a fixed number of common factors does not strongly affect our estimation results. Consequently, in deference to the principle of parsimony, we argue that the simpler model is to be preferred.

Additional output from the model estimated using a time-varying number of common factors is available from the authors on request.

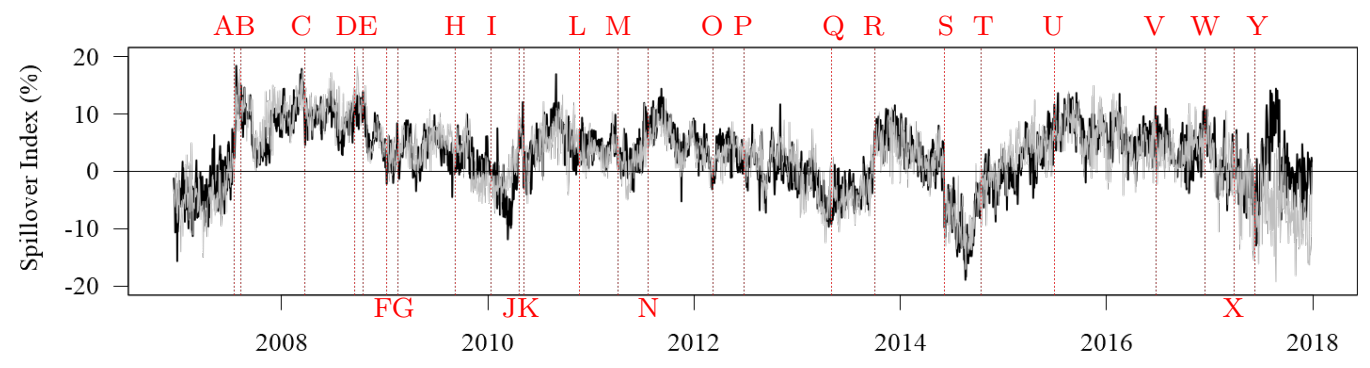

\begin{abstract}
Notes: The heavy black line shows our baseline estimate of the $5 \%$ RTD. The gray line shows the $5 \%$ RTD obtained allowing the number of common factors to vary across rolling samples. The results are obtained from rolling regressions with a window length of 250 trading days using a forecast horizon of 5 trading days. The date shown corresponds to the last day of each rolling window. We suppress rolling samples where the system exhibits instability at the conditional mean. The following events are marked: A: Bear Stearns announces hedge fund losses (17-Jul-07); B: BNP Paribas halts redemptions on three investment funds (09-Aug-07); C: Bear Stearns is acquired by JP Morgan (24-Mar-08); D: Lehman Brothers files for bankruptcy (15-Sep-08); E: the Troubled Asset Relief Program is announced (14-Oct-08); F: the Fed begins purchasing mortgage-backed securities issued by Fannie Mae and Freddie Mac (05-Jan-09); G: signing of the American Recovery and Reinvestment Act (17-Feb-09); H: Greek parliament is dissolved (08-Sep-09); I: report on the falsification of Greek data released (12-Jan-10); J: Greece requests aid (23-Apr-10); K: the European Financial Stability Facility is announced (09May-10); L: Ireland requests aid (22-Nov-10); M: Portugal requests aid (06-Apr-11); N: second Greek bailout (22-Jul-11); O: Greek debt swap arrangement agreed (07-Mar-2012); P: Spain requests aid (25-Jun-2012); Q: ECB cuts interest rates to a record low of 0.5\% (01-May-2013); R: US government shutdown (01-Oct-2013); S: ECB announces negative interest rate policy (05-Jun-2014); T: October 2014 flash crash (15-Oct-2014); U: Greece fails to meet its IMF payment schedule (30-Jun-2015); V: Brexit referendum (23-Jun-2016); W: Federal Reserve raises interest rates (14-Dec-2016); X: UK invokes article 50 of the Lisbon Treaty (29-Mar-2017); and Y: snap election held in the UK (08-Jun-2017).
\end{abstract}

Figure B1: Robustness of RTD to Time-Variation in the Number of Common Factors 


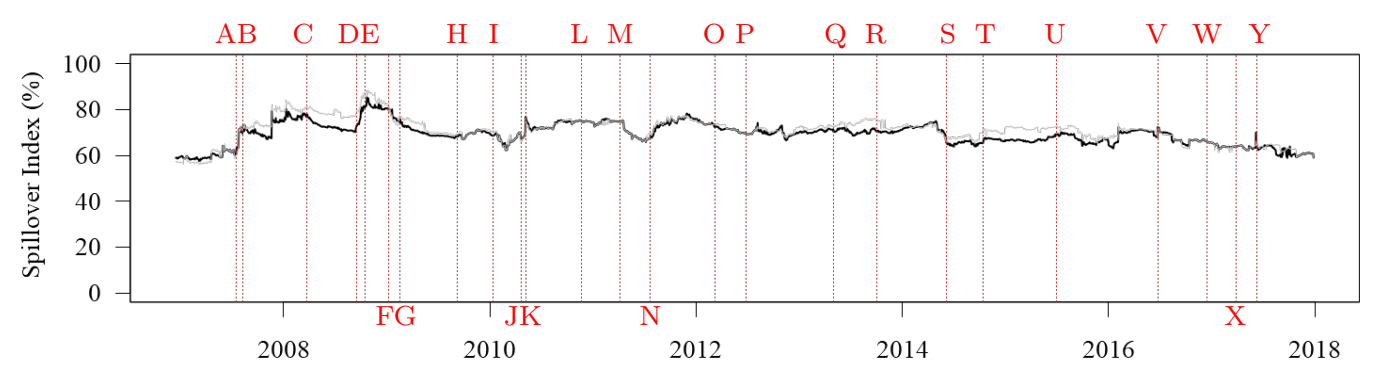

(a) Conditional Mean

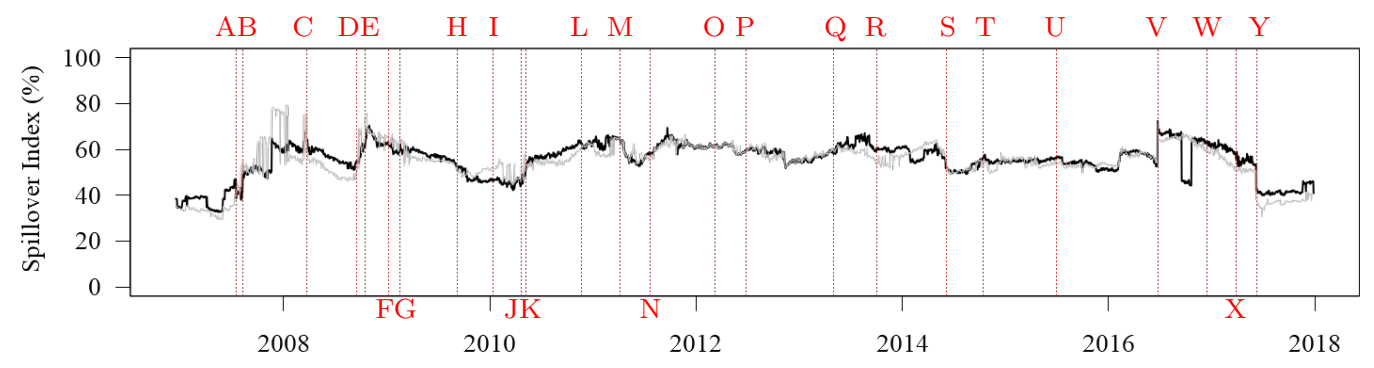

(b) Conditional Median

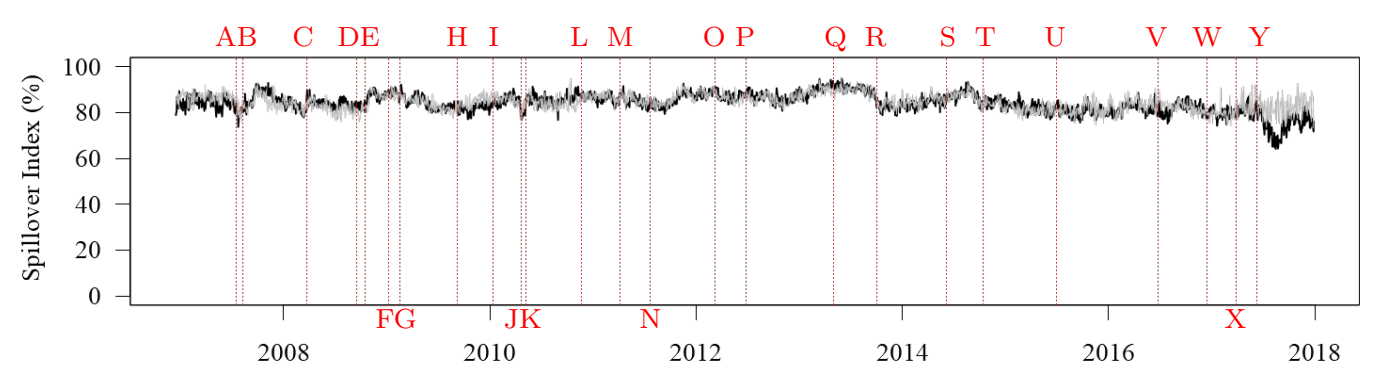

(c) Fifth Conditional Quantile

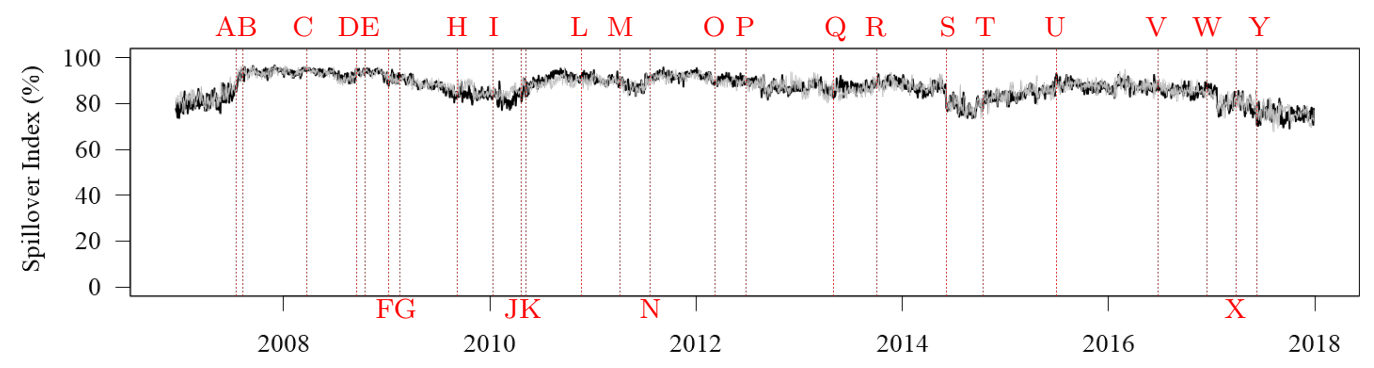

(d) Ninety-Fifth Conditional Quantile

Notes: The heavy black line in each panel shows our baseline estimate of the DY spillover index. The gray line shows the DY spillover index obtained allowing the number of common factors to vary across rolling samples. The results are obtained from rolling regressions with a window length of 250 trading days using a forecast horizon of 5 trading days. The date shown corresponds to the last day of each rolling window. We suppress rolling samples where the system exhibits instability at the conditional mean. The following events are marked: A: Bear Stearns announces hedge fund losses (17-Jul-07); B: BNP Paribas halts redemptions on three investment funds (09-Aug-07); C: Bear Stearns is acquired by JP Morgan (24-Mar-08); D: Lehman Brothers files for bankruptcy (15-Sep-08); E: the Troubled Asset Relief Program is announced (14-Oct-08); F: the Fed begins purchasing mortgage-backed securities issued by Fannie Mae and Freddie Mac (05-Jan-09); G: signing of the American Recovery and Reinvestment Act (17-Feb-09); H: Greek parliament is dissolved (08-Sep-09); I: report on the falsification of Greek data released (12-Jan-10); J: Greece requests aid (23-Apr-10); K: the European Financial Stability Facility is announced (09-May-10); L: Ireland requests aid (22-Nov-10); M: Portugal requests aid (06-Apr-11); N: second Greek bailout (22-Jul-11); O: Greek debt swap arrangement agreed (07-Mar-2012); P: Spain requests aid (25-Jun-2012); Q: ECB cuts interest rates to a record low of 0.5\% (01-May-2013); R: US government shutdown (01-Oct-2013); S: ECB announces negative interest rate policy (05-Jun-2014); T: October 2014 flash crash (15-Oct-2014); U: Greece fails to meet its IMF payment schedule (30-Jun-2015); V: Brexit referendum (23-Jun-2016); W: Federal Reserve raises interest rates (14-Dec-2016); X: UK invokes article 50 of the Lisbon Treaty (29-Mar-2017); and Y: snap election held in the UK (08-Jun-2017).

Figure B2: Robustness to Time-Variation in the Number of Common Factors 


\title{
Appendix C: Financial Institutions Included in the Synthetic Fi- nancial Sector CDS Spread Indices, by Country
}

The financial institutions that are included in the synthetic financial sector CDS spreads developed by Greenwood-Nimmo et al. (2019) for each country are listed below.

\begin{abstract}
Australia
Adelaide Bank, AMP, Australia and New Zealand Banking Group, Bendigo and Adelaide Bank, Bank of Queensland, Commonwealth Bank of Australia, Insurance Australia Group, Macquarie Group, National Australia Bank, QBE Insurance Group, St. George Bank, Suncorp Group, and Westpac Banking Corporation.
\end{abstract}

Austria

Erste Group Bank, and Raiffeisen Zentralbank.

Belgium

Ageas, Fortis and KBC Groep.

China

Agricultural Bank of China, Bank of China, Bank of Communications, and Industrial and Commercial Bank of China.

France

Credit Agricole, Axa, BNP Paribas, Natixis, Scor, and Société Générale.

\section{Germany}

Allianz, Commerzbank, Deutsche Bank, Deutsche Postbank, Hannover RE, IKB Deutsche Industriebank, Munich RE, and Talanx.

\section{Ireland}

Allied Irish Banks, Anglo Irish Bank, Bank of Ireland, Irish Bank Resolution Corporation, and Permanent TSB.

\section{Italy}

Generali Assicuraziono, Mediobanca, Banca Italease, Banco Popolare di Verona e Novara, Banca Popolare Italiana, Banco Popolare, Banca Lombarda e Piemontese, Capitalia, San Paolo IMI, Banca Popolare di Milano SCARL, Banca Monte dei Paschi di Siena, Intesa Sanpaolo, Unione di Banche Italiane, Unipol Gruppo Finanziario, and Unicredit.

\section{Japan}

ACOM Company, Aeon Financial Service Company, Aozora Bank, Bank of Fukuoka, Bank of Iwate, Bank of Yokohama, Chiba Bank, Credit Saison Company, Dai-Ichi Life Insurance Company, Daiwa Securities Group, Higo Bank, Hiroshima Bank, Hitachi Capital Corporation, Hyakugo Bank, Joyo Bank, MS\&AD Insurance Group Holdings, Mizuho Holdings, Mizuho Trust and Banking Company, Mitsubishi UFJ Financial Group, Nishi-Nippon City Bank, Nikko Cordial Corporation, Nomura Holdings, Orix Corporation, Resona Bank, The 77 Bank, Shiga Bank, Shinsei Bank, Shizuoka Bank, Sompo Japan Insurance, Sumitomo Mitsui Financial Group, Sumitomo Mitsui Trust Bank, and Tokio Marine \& Nichido Fire Insurance Company.

\section{Netherlands}

ABN Amro Bank, Aegon, Ageas, Fortis, ING Groep, NN Group, SNS Reaal, and van Lanschot.

\section{Norway}

DNB, and Storebrand Group.

\section{Portugal}

Banco BPI, Banco Commercial Portugues, Banco Espirito Santo, and Novo Banco. 


\section{Russian Federation}

Bank of Moscow, Bank Uralsib, Gazprombank, MDM Bank, Promsvyazbank, Sberbank of Russia, and VTB Bank.

\section{Spain}

Bankia, Banco de Sabadell, Banco Bilbao Vizcaya Argentaria, Bankinter, Banco Popular Espanol, Banco Pastor, and Banco Santander.

\section{Sweden}

Nordea Bank, Skandinaviska Enskilda Banken, Svenska Handelsbanken, and Swedbank.

\section{United Kingdom}

Aviva, Barclays, Bradford \& Bingley, Friends Provident, HBOS, HSBC Holdings, 3I Group, Legal \& General Group, Lloyds Banking Group, Man Strategic Holdings, Old Mutual, Prudential, Royal Bank of Scotland Group, RSA Insurance Group, Standard Life, and Standard Chartered.

\section{United States}

Aflac Inc., Allstate Corporation, Ally Financial Inc., American Express Company, American Financial Group Inc., American International Group Inc., Assurant Inc., Bank of America Corporation, Bank of New York Company Inc., Bank of New York Mellon Corporation, BB\&T Corporation, Bear Stearns Companies Inc., Berkshire Hathaway Inc., Blackrock Inc., Capital One Financial Corporation, Charles Schwab Corporation, Chubb Corporation, CIT Group Inc., Citigroup Inc., CNA Financial Corporation, Discover Financial Services, E*Trade Financial Corporation, Federal National Mortgage Association, Fifth Third Bancorp, Franklin Resources Inc., Genworth Holdings Inc., Goldman Sachs Group Inc., Hartford Financial Services Group Inc., Huntington National Bank, Jefferies Group LLC, JP Morgan Chase \& Company, Keycorp, Legg Mason Inc., Lehman Brothers Holdings Inc., Lincoln National Corporation, Loews Corporation, Markel Corporation, Marshall \& Ilsley Corporation, MBIA Inc., Mellon Financial Corporation, Merrill Lynch \& Company Inc., Metlife Inc., Morgan Stanley, National City Corporation, Nationwide Financial Services, PHH Corporation, PNC Financial Services Group Inc., Principal Financial Group Inc., Progressive Corporation, Protective Life Corporation, Prundential Financial Inc., Regions Financial Corporation, Reinsurance Group of America Inc., SLM Corporation, State Street Corporation, Suntrust Banks Inc., Torchmark Corporation, Travelers Companies Inc., Unum Group Inc., US Bancorp, WR Berkeley Corporation, Wachovia Corporation, and Wells Fargo \& Company. 


\section{Appendix D: Details of the Observed Global Variables}

The observed global variables used in our analysis of the properties of the unobserved factors in Section 4.1 are defined as follows:

(i) US stock market performance. To capture the key risk factors affecting US equity markets, we use the Rm-Rf, SMB and HML factors developed by Fama and French (1993). RmRf represents a market factor, while SMB and HML account for risk factors related to firm size and book-to-market equity, respectively. The Fama-French factors are freely available from Ken French via http://mba.tuck. dartmouth.edu/pages/faculty/ken. french/data_library.html.

(ii) US Treasury market conditions. We use the change in the five-year Constant Maturity Treasury (CMT) yield to capture expectations regarding macroeconomic conditions in the US and, by extension, in the world economy. In addition, given that investors regard US Treasury debt as a safe haven asset, Longstaff et al. (2011) note that the CMT yield may incorporate a flight-to-liquidity component. The CMT yield is published by the Federal Reserve in its H.15 Statistical Release.

(iii) The TED spread. The TED spread is the difference between the 3-month USD LIBOR and the 3-month US Treasury bill yield. Variations in the TED spread reflect changes in counterparty risk and liquidity in the US interbank market. Consequently, it is widely used as an indicator of funding liquidity. The TED spread is available from the Federal Reserve Economic Data Service (FRED) via https://fred.stlouisfed.org/.

(iv) The Euribor-DeTBill spread. To capture European-specific variations in funding liquidity, we use the spread between the 3-month Euribor and the 3-month German Treasury bill yield. The Euribor and the German yield data are available from Datastream.

(v) The variance risk premium (VRP). Bollerslev, Tauchen and Zhou (2009) define the VRP as the difference between the one-month-ahead implied variance and a forecast of the realized variance over the same period. Under this definition, the VRP is typically positive, with higher values indicating a reduced risk appetite. We forecast the realized variance using the same augmented version of Corsi's (2009) heterogeneous autoregressive model used by Bekaert and Hoerova (2014). We compute the VRP as $V R P_{t}=V I X_{t}^{2}-E\left[R V_{t+1}^{(22)}\right]$, where $V I X_{t}^{2}$ denotes the de-annualized squared VIX and $R V_{t}^{(22)}$ denotes the realized variance for the S\&P 500 measured over the next 22 trading days as the sum of squared five-minute intraday returns. The VIX data is available from FRED, while we obtain the daily realized variance from the Oxford Man Institute's Realized Library (Heber, Lunde, Shephard and Sheppard, 2009, ver. 0.2). ${ }^{15}$

(vi) The Treasury term premium. The term premium measures the excess yield required to induce investors to hold a long-term bond as opposed to a sequence of shorter-term bonds. Consequently, it conveys valuable information on investors' time preferences as well as their expectations regarding the macroeconomic outlook. We use the 5-year Treasury term premium derived from the five-factor no-arbitrage term structure model of Adrian, Crump and Moench (2013), which is freely available from the Federal Reserve Bank of New York via https://www.newyorkfed.org/research/data_indicators/term_premia.html.

\footnotetext{
${ }^{15}$ Longstaff et al. (2011) also consider the equity premium approximated at monthly frequency by the priceearnings ratio for the S\&P 100 index. We are obliged to exclude the equity premium, because earnings per share is unavailable at daily frequency. However, the variance risk premium should be highly correlated with the equity premium and should impound much of its informational content.
} 
(vii) US investment grade and high yield spreads. To capture changes in the required rate of return on investment grade (IG) and high yield (HY) corporate bonds, we use both the IG and HY spreads. We define the IG spread as the spread between the Bank of America Merrill Lynch US corporate BBB and AAA effective yields and the HY spread as the difference between the Bank of America Merrill Lynch US corporate BB and BBB effective yields. The corporate bond yield data is available from FRED.

(viii) ITRAXX indices to capture pan-European credit risk factors. To account for European credit risk factors not captured elsewhere, we use five 5-year ITRAXX indices to isolate European credit risk factors. Specifically, we include the ITRAXX Europe index, the ITRAXX High Volatility index, ITRAXX Crossover index and the ITRAXX Senior and Subordinated Financials indices. ITRAXX data is available from Datastream.

(ix) Bilateral spot exchange rate returns. To capture exchange rate fluctuations, we use the daily log-return on the bilateral spot exchange rate for each currency in our sample in units of foreign currency per USD. The exchange rate data is obtained from Datastream. 


\section{University Library}

\section{- M M N E R VA A gateway to Melbourne's research publications}

Minerva Access is the Institutional Repository of The University of Melbourne

Author/s:

Ando, T;Greenwood-Nimmo, M;Shin, Y

Title:

Quantile Connectedness: Modeling Tail Behavior in the Topology of Financial Networks

Date:

2022-04-01

Citation:

Ando, T., Greenwood-Nimmo, M. \& Shin, Y. (2022). Quantile Connectedness: Modeling Tail Behavior in the Topology of Financial Networks. MANAGEMENT SCIENCE, 68 (4), pp.2401-2431. https://doi.org/10.1287/mnsc.2021.3984.

Persistent Link:

http://hdl.handle.net/11343/258634 